\title{
Analytical and numerical analysis on the collapse modes of least- thickness circular masonry arches at decreasing friction
}

Giuseppe Cocchetti

Politecnico di Milano, Dipartimento di Ingegneria Civile e Ambientale,

piazza L. da Vinci 32, I-20133 Milano, Italy

giuseppe.cocchetti@polimi.it, bttp:/ / orcid.org/0000-0002-9695-2967

\section{Egidio Rizzi*}

Università degli studi di Bergamo, Dipartimento di Ingegneria e Scienze Applicate, viale G. Marconi 5, I-24044 Dalmine (BG), Italy

egidio.rizzi@unibg.it, bttp://orcid.org/0000-0002-6734-1382

AвSTRACT. Departing from pioneering Heyman modern rational investigations on the purely-rotational collapse mode of least-thickness circular masonry arches, the hypothesis that joint friction shall be high enough to prevent inter-block sliding is here released. The influence of a reducing Coulomb friction coefficient on the collapse modes of the arch is explicitly inspected, both analytically and numerically, by tracing the appearance of purely-rotational, mixed sliding-rotational and purely-sliding modes. A classical doubly built-in, symmetric, complete semi-circular arch, with radial joints, under self-weight is specifically considered, for a main illustration. The characteristic values of the friction coefficient limiting the ranges associated to each collapse mode are first analytically derived and then numerically identified, by an independent self-implementation, with consistent outcomes. Explicit analytical representations are provided to estimate the geometric parameters defining the limit equilibrium states of the arch, specifically the minimum thickness to radius ratio, at reducing friction. These formulas, starting from the analysis of classical Heymanian instance of purely-rotational collapse, make new explicit reference to the mixed slidingrotational collapse mode, arising within a narrow range of limited friction coefficients (or friction angles). The obtained results are consistently compared to existing numerical ones from the competent literature. ${ }^{1}$

KEYWORDS. Circular masonry arches; Couplet-Heyman problem; Reducing friction; Purely-rotational mode; Mixed sliding-rotational mode; Purely-sliding mode.

\section{OPEN ACCESS}

Citation: Cocchetti, G., Rizzi, E., Analytical and numerical analysis on the collapse modes of least-thickness circular masonry arches at decreasing friction, Frattura ed Integrità Strutturale, 51 (2020) 356-375.

Received: 30.06 .2019

Accepted: 05.11.2019

Published: 01.01.2020

Copyright: (C) 2020 This is an open access article under the terms of the CC-BY 4.0, which permits unrestricted use, distribution, and reproduction in any medium, provided the original author and source are credited.

\footnotetext{
${ }^{1}$ An earlier version of the present work with preliminary developments was presented at the SAHC12 conference in Wroclaw, Poland, October 15-17, 2012 [7].
} 


\section{INTRODUCTION}

$\mathrm{F}$ irst rational studies on the statics of masonry arches were blooming in 1700 , with the development of so-called pre-elastic theories. In recent times, they have culminated, in the second half of 1900, in the modern reinterpretation and application of Limit Analysis to masonry constructions, according to the fundamental pioneering work by Jacques Heyman [1-4].

Specifically, Heyman stated three classical behavioural assumptions for masonry structures (1 - no tensile strength; 2 - infinite compressive strength; 3 - no sliding failure), and investigated the five-hinge purely-rotational collapse mode of continuous symmetric circular masonry arches under self-weight (taken as uniformly-distributed along the geometrical centreline of the arch, with self-weight per unit length $w=\gamma t d$ ), by providing analytical representations for the determination of the characteristic parameters of the masonry arch in the critical, least-thickness condition, such as (Figs. 1a,b):

- angular position $\beta$ of the rupture (radial) joint with inner intrados hinge at the haunches;

- critical thickness to radius ratio $\eta=t / r$;

- non-dimensional horizontal thrust $b=H /(w r)$ acting in such a limit state of minimum thickness still available to sustain the arch (Couplet-Heyman problem).

This classical least-thickness problem in the statics of masonry arches has been revisited by the present authors within a wide research project that has been considering different characteristic aspects, by employing both self-consistent analytical and numerical techniques [5-10]. Arches of a general half-angle of embrace $0<\alpha<\pi$ (including for undercomplete and over-complete, horseshoe circular masonry arches) have been systematically analysed in analytical terms. Different solutions have been explicitly derived, and numerically explored, which appeared fully consistent with updated outcomes from a re-discussion by Heyman [4], and prior developments by Ochsendorf [11-12], as well as with classical earlier work by Milankovitch [13] (see Foce [14]), and several most recent attempts that meanwhile have appeared [15-24]. An earlier account on these developments was provided in SAHC10 conference paper [5]; later, a comprehensive analytical treatment with unprecedented closed-form explicit representations was provided in [6], while in [8], consistent comparisons were developed by a Discrete Element Method implementation, in the form of a Discontinuous Deformation Analysis (DDA) tool.

Then, first new developments on the role of friction have been preliminarily investigated in such a research mainstream, as initially reported in SAHC12 conference work [7], prodromal to the present one, by releasing Heyman hypothesis 3 of no sliding failure, and accounting for both mixed sliding-rotational and purely-sliding collapse modes (Figs. 1c,d). Here, within that context, a new, complete, analytical treatment is developed, starting from the developed analytical solutions that had earlier been derived for purely-rotational collapse [6,8], with additional comprehensive numerical verification. Very recently, separate innovative numerical implementations as an optimisation problem to be solved by non-linear Mathematical Programming are being developed [9,10], with results that turn out truly consistent with the ones here derived and presented.

Specifically, the limit values of Coulomb friction coefficient $\mu=\tan \varphi$ (and friction angle $\varphi$ ), at the theoretical (radial) joints of a continuous arch, marking the transitions between the three collapse modes depicted in Figs. 1b-d are here explicitly derived, together with the determination of the analytical dependence of the mixed-mode collapse characteristics (Fig. 1c), as a function of friction coefficient $\mu$, namely $\beta_{m}(\mu), \eta_{m}(\mu), h_{m}(\mu)$.

It is newly shown that, at decreasing friction coefficient $\mu$ at the (radial) joints of the arch, horizontal thrust $h_{m}(\mu)$ under mixed mode becomes fixed (decreasing), by finite (reducing) friction. This induces, as a consequence, non-linear increasing dependencies of $\eta(\mu)$ and $\beta(\mu)$. In other words, at a decreasing friction coefficient at the joints of the arch, an increase in the critical value of least thickness is required to warrant the equilibrium of the self-standing masonry arch.

Here, a main reference is made to complete semi-circular arches with a $2 \alpha=\pi$ opening (Fig. 1a), for a comprehensive illustration and discussion. For such a reference case, the friction range in which mixed-mode collapse is shown to appear turns out quite narrow, in practical terms, i.e. with friction angles $\varphi$ between around $22^{\circ}$ and $17^{\circ}(\mu=\tan \varphi$ between around 0.4 and 0.3 ).

Despite, that the value of these findings shall appear to be more of a theoretical type (complete behaviour of a codified mechanical system), than of a practical nature (usually friction coefficients in masonry constructions shall safely be above that; arches are normally built with much higher margins in terms of thickness to radius ratios, with respect to the critical least-thickness condition; and so on), conditions that may be associated to a reducing friction, such as loosening joints, insertion of external materials among the blocks, non-firm or spreading supports, etc., may come up into the picture, leading to considerable interest in anyway reaching a full understanding on arch "stability" (say, equilibrium) at reducing 
friction, as in the present setting. Moreover, the critical value of friction coefficient up to induce possible sliding may increase with the opening angle of the arch (specifically for over-complete horseshoe arches, see later discussion in Section 2.3), thus possibly approaching the ranges of friction coefficients that may be encountered in practice and maybe leading to the potential appearance of sliding within the failure mode.

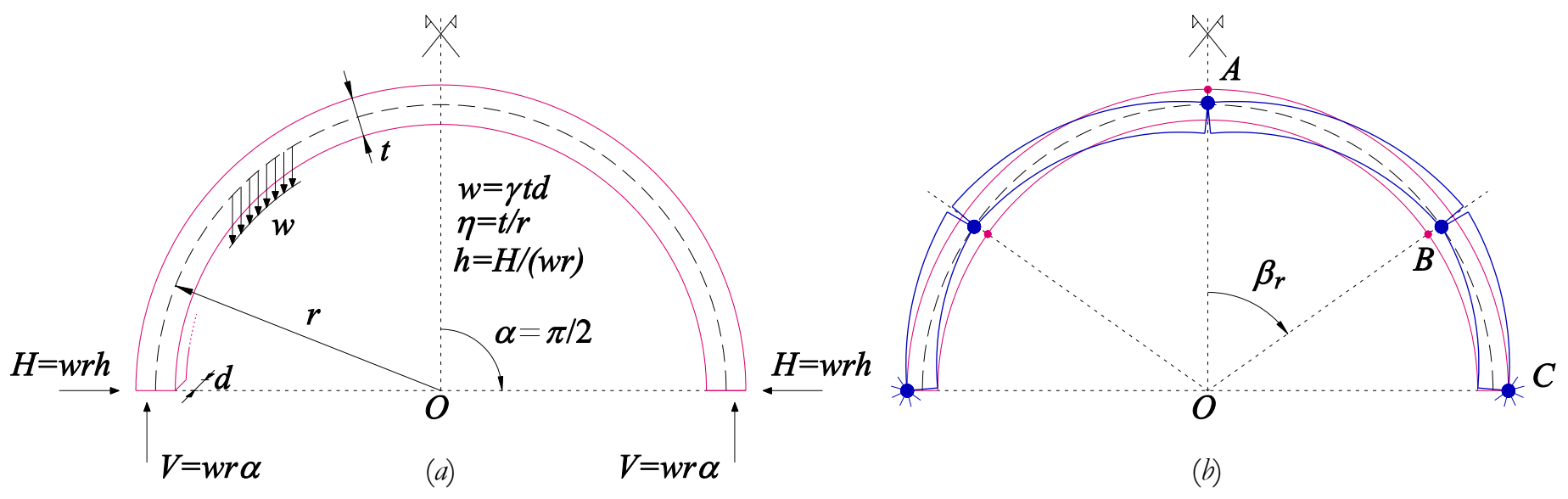

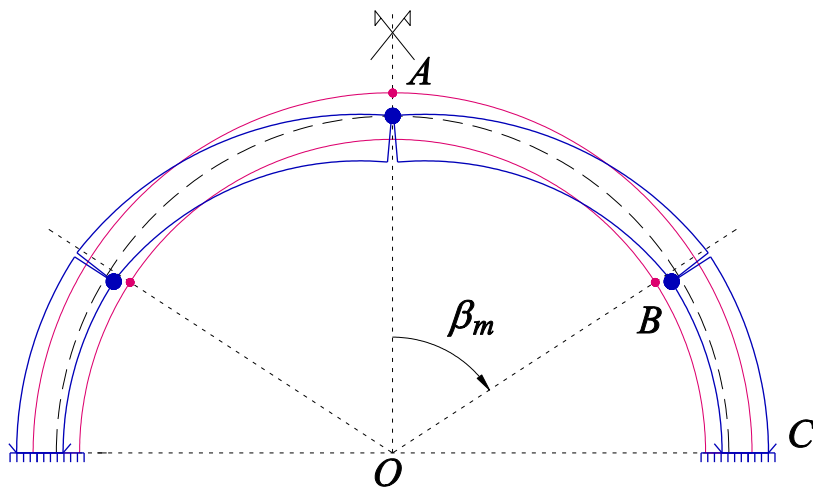

(c)

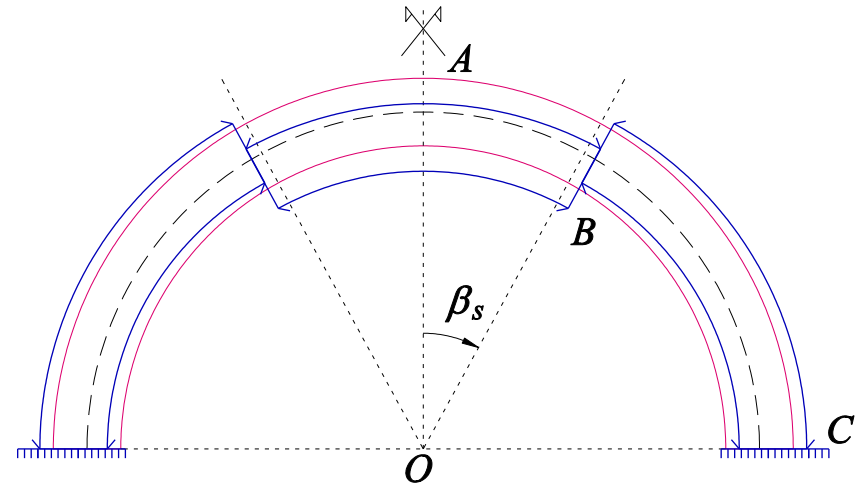

(d)

Figure 1: Sketches of a symmetric semi-circular masonry arch under self-weight: (a) characteristic parameters, primarily: half-angle of embrace $\alpha$, inner rupture joint angular position $\beta$, thickness to radius ratio $\eta$, non-dimensional horizontal thrust $b$;

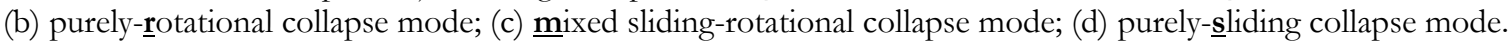

The present analytical and numerical results appear to be consistent to ones numerically developed in the existing literature, specifically concerning the effects of friction in masonry arches. For instance, Gilbert et al. [25] have numerically investigated the role of friction, by estimating for a semi-circular arch a minimum thickness to radius ratio $\eta=0.1068$, in the presence of a purely-rotational collapse mode, when $\mu$ is greater than 0.396 (friction angle larger than $\left.\varphi=21.60^{\circ}\right)$. This marks, at decreasing friction, the transition from the purely-rotational mode to the mixed slidingrotational mode. Furthermore, a value of $\mu=0.31\left(\varphi=17.22^{\circ}\right)$ was found, which then located the shift from the mixed sliding-rotational mode to the purely-sliding mode. In Gilbert et al. [25] a characteristic, sort of L-shaped diagram depicts the critical value of thickness to radius ratio $\eta$ as a function of friction coefficient $\mu$, with a double kink at these two critical values of $\mu$, a constant value of $\eta$ for $\mu>0.396$ and rapidly-growing values of $\eta$ right on $\mu=0.31$. These outcomes appear in good agreement with numerically developed earlier results by Sinopoli et al. [26-28], which individuated the above-mentioned kinks respectively at $\mu=0.395\left(\varphi=21.55^{\circ}\right)$ and $\mu=0.309\left(\varphi=17.17^{\circ}\right)$.

Here, the following "exact" analytical results for the complete semi-circular arch are going to be consistently derived: $\mu_{r m}=0.395832\left(\varphi_{r m}=21.5952^{\circ}\right)$, for the transition from purely-rotational to mixed sliding-rotational modes; $\mu_{m s}=0.309215\left(\varphi_{m s}=17.1824^{\circ}\right)$, for the transition between mixed and purely-sliding modes. 
In the present paper, two sorts of analyses on the role of reducing friction in circular masonry arches are carried-out, as respectively presented in Sections 2 and 3, with mutually consistent results, as eventually outlined in resuming Section 4:

- First, a complete analytical approach in the wake of previous original analytical solutions for purely-rotational collapse [6,8] is developed, towards the characterisation of the mixed sliding-rotational collapse mode and of the relevant friction bounds. This analytical analysis starts from the presumed purely-rotational collapse mode due to Heyman [1-4] and, by decreasing friction, derives, through a static approach, the modes that subsequently arise, with explicit "exact" solutions.

- Second, a comprehensive, original self-implemented numerical approach is developed, which sets an optimisation analysis in the sense of the lower-bound (static) theorem of Limit Analysis, within a classical commercial spreadsheet wherein an optimisation function is made available. There, the constitutive behaviour of the circular masonry arch is stated and equilibrium conditions are varied towards the determination of the critical least-thickness condition and of the attached collapse mode. Thus, the collapse mode, whether purely-rotational, mixed sliding-rotational or purely-sliding, is not there a priori hypothesised, while correctly numerically recovered, through an optimisation process, with outcomes that turn out fully coherent with those "exact" ones from the previous analytical analysis, and also to the above-quoted findings from the preceding literature [25-28].

\section{ANALYTICAL APPROACH}

$\mathrm{S}$

tarting from the classical analysis of purely-rotational collapse of circular masonry arches in the so-called Couplet-Heyman problem [1-4], the determination of collapse characteristics $\beta, \eta, b$ for a symmetric circular arch of general half-angle of embrace $\alpha$ (Figs. 1a,b) may be stated by the solution of the following system of three characteristic equations, in terms of unknown non-dimensional horizontal thrust variable $h[5,6,8]$ :

$$
\left\{\begin{array}{l}
b=h_{1}=\frac{(2-\eta) \beta \sin \beta-2(1-\cos \beta)\left(1+\delta_{M} \eta^{2} / 12\right)}{2+\eta-(2-\eta) \cos \beta} \\
b=h_{2}=A-\frac{2}{2+\eta}\left(1+\delta_{M} \eta^{2} / 12\right), \quad A=\alpha \cot \frac{\alpha}{2} \\
b=h_{e}=\frac{(2-\eta)(\sin \beta+\beta \cos \beta)-2 \sin \beta\left(1+\delta_{M} \eta^{2} / 12\right)}{(2-\eta) \sin \beta}=\underbrace{\beta \cot \beta}_{h_{H}}-\frac{\eta}{2-\eta} \delta_{C C R}\left(1+\delta_{M} \eta / 6\right)
\end{array}\right.
$$

Eqns. (1) and (1) $)_{b}$ represent two equilibrium relations, respectively the rotational equilibrium of any upper portion $A B$ of the half-arch (symmetry conditions apply) with respect to inner intrados hinge at haunch $\mathrm{B}$, and of whole half-arch AC with respect to extrados hinge at shoulder $\mathrm{C}$ (Fig. 1b); the latter introduces the dependence on opening angle $\alpha$, through variable $A=\alpha \cot (\alpha / 2)$.

Importantly, Eqn. (1) ctruly corresponds to the correct tangency condition of the line of thrust (locus of pressure points) at haunch intrados B and may be derived from the following stationary condition:

$$
h_{1}^{\prime}(\beta)=\frac{d h_{1}(\beta)}{d \beta}=0 \quad \Rightarrow \quad b=h_{e}=\frac{\operatorname{num}\left[h_{1}\right]^{\prime}}{\operatorname{den}\left[b_{1}\right]^{\prime}}
$$

where symbol ( )' denotes a differentiation with respect to $\beta[6]$ and advantage has been taken from the quotient rule of differentiation.

Notice that the correct statement of this tangency condition actually sets a main difference to classical Heyman solution, which stated the tangency condition on the thrust force itself ( $b=h_{H}$ in Eqn. (1)c), and may be considered as leading to a sort of approximation of the true solution, at least until when $\eta$ keeps small.

Moreover, in Eqns. (1) $\delta_{C C R}$ and $\delta_{M}$ are two control flags allowing to shift from classical Heyman solution $\left(\delta_{C C R}=0\right.$, $\left.\delta_{M}=0\right)$, to the correct solution of Heyman problem $\left(\delta_{C C R}=1, \delta_{M}=0\right)$, and to Milankovitch solution considering the true 
self-weight distribution along the arch $\left(\delta_{C C R}=1, \delta_{M}=1\right)$, respectively leading to a "linear", a "quadratic" and a "cubic problem" in the algebraic solution for unknown triplet $A(\beta), \eta(\beta), b(\beta)$. This is originally and extensively derived, described and discussed in [6].

All what will be considered in the following will stick to classical hypothesis $\delta_{M}=0$, i.e. by assuming a self-weight distribution along the geometrical centreline of the arch, in classical Heyman sense, though with correct evaluation $\delta_{C C R}=1$ of the tangency condition in Eqn. (1)c. An extensive discussion on the differences between the three arising solutions has been reported in [6], including about the spreading discrepancies appearing for over-complete (horseshoe) circular masonry arches.

Known Heyman "linear" solution may finally be obtained from Eqns. (1), for $\delta_{C C R}=0, \delta_{M}=0$, and can be classically represented as:

$$
\left\{\begin{array}{l}
A=\alpha \cot \frac{\alpha}{2}=\beta \cot \beta \frac{2 \beta \cos \beta+\sin \beta \cos ^{2} \beta+\sin \beta}{2 \beta \cos \beta+\sin \beta \cos ^{2} \beta-\sin \beta \cos \beta} \\
\eta=\eta_{H}=2 \frac{(\beta-\sin \beta)(1-\cos \beta)}{\beta(1+\cos \beta)} \\
b=b_{H}=\beta \cot \beta
\end{array}\right.
$$

Going to properly correct CCR solution $\left(\delta_{C C R}=1, \delta_{M}=0\right)$, for the complete semi-circular arch, i.e. $\alpha=A=\pi / 2$, system (1) renders the following characteristic CCR solution triplet for the purely-rotational collapse mode to be recorded:

$$
\beta_{r}=0.951141 \mathrm{rad}=54.4963^{\circ}, \quad \eta_{r}=0.107426, \quad h_{r}=0.621772 \quad(\alpha=\pi / 2)
$$

More generally, at variable half-opening angle $\alpha$ of the circular masonry arch (thus at variable $A=\alpha \cot (\alpha / 2)$ ), the solution of system (1) for $\delta_{C C R}=1, \delta_{M}=0$ can be analytically represented in closed-form as follows [6]:

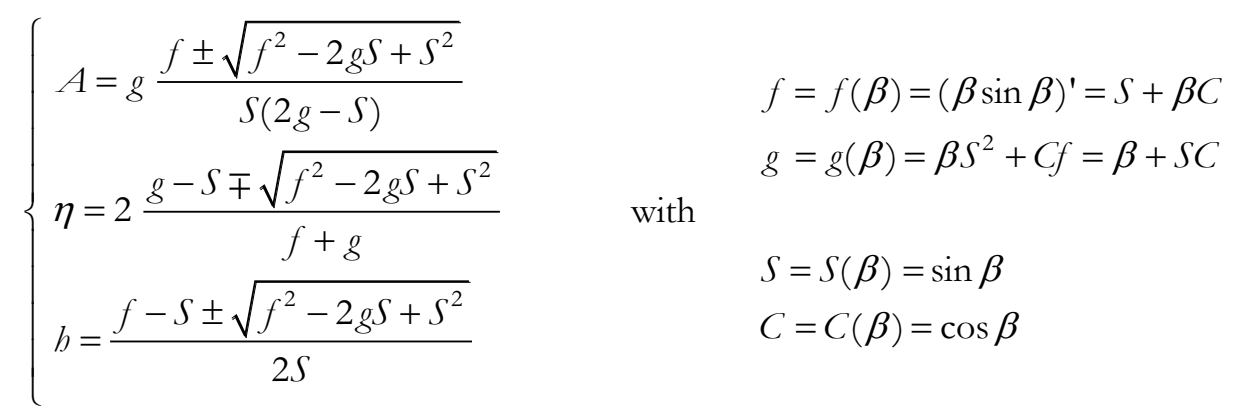

Indeed, triplet $A(\beta), \eta(\beta), b(\beta)$ becomes a double-valued function of inner hinge position $\beta$, coming from the solution of the following "quadratic problem" [6]:

$$
\left\{\begin{array}{l}
S(2 g-S) A^{2}-2 f g A+g^{2}=0 \\
(f+g) \eta^{2}-4(g-S) \eta+4(g-f)=0 \\
2 S b^{2}-2(f-S) b+g-f=0
\end{array}\right.
$$

The above three quadratic equations in $A, \eta, b$ can be obtained from system (1) by eliminating in turn couples $(\eta, b)$, $(A, b)$ and $(A, \eta)$. Notice that solutions $(5)_{\mathrm{b}}$ and $(5)_{\mathrm{c}}$ for $\eta$ and $h$ can be obtained from a $2 \times 2$ subsystem formed by Eqns. (1)a and (1)c, namely those independent on $A(\alpha)$ in source system (1). Functions $A(\beta), \eta(\beta), b(\beta)$ become single-valued at $\beta=\beta_{s \beta}=1.12909 \mathrm{rad}=64.6918^{\circ}$, namely at the value of $\beta$ setting to zero the term under square roots 
in Eqns. (5): $f^{2}-2 g S+S^{2}=0$. This corresponds to a stationary (maximum) point of the curves $\beta(\alpha)$ or $\beta(A)$ at variable arch opening [6,8]. The characteristic solution for $\alpha=A=\pi / 2$ keeps in the pre-peak branch $\left(A^{+}, \eta, b^{+}\right)$of solution (5).

Solutions $(5)_{\mathrm{a}},(5)_{\mathrm{b}}$ and $(5)_{\mathrm{c}}$ can be analytically plotted as a function of hinge position $\beta$, as depicted in Fig. 2, with comparison between correct CCR solution [6] and classical (say "approximated") Heyman solution.

Similar representations occur as well for Milankovitch solution accounting for the true self-weight distribution along the circular arch, though leading to the following more involved "cubic problem":

$$
\left\{\begin{array}{l}
S^{2}(3 g-2 S) A^{3}+3(g-f) g S A^{2}-3 f g^{2} A+2 g^{3}=0 \\
S \eta^{3}+3(f+g) \eta^{2}-12(g-S) \eta+12(g-f)=0 \\
6 S^{2} b^{3}-3 S(3 f-g-2 S) b^{2}-3(g-f)(f-2 S) b+2(g-f)^{2}=0
\end{array}\right.
$$

with rather similar results and minor differences, to those recovered for the above "quadratic problem" [6]. Thus, the value of Milankovitch solution is not further inspected here, since focus is now going to be made on the effect of reducing friction, on correct CCR solution, vs. "approximated" Heyman one, in the kept hypothesis of self-weight distribution along geometrical centreline of the circular arch.

Moreover, CCR solutions (5) $)_{\mathrm{b}}$ and (5) can be analytically plotted by parametric plots $(\eta(\beta), h(\beta))$ and $(\beta, h(\beta))$, for $0 \leq \beta \leq \beta_{s \beta}$, showing (with important implications in the present forthcoming analysis on the role of reducing friction) non-linear dependencies of $\eta(b)$ and $\beta(b)$ at variable non-dimensional horizontal thrust $b$ (Figs. 3a,b), in the critical condition of purely-rotational collapse (Fig. 1b).
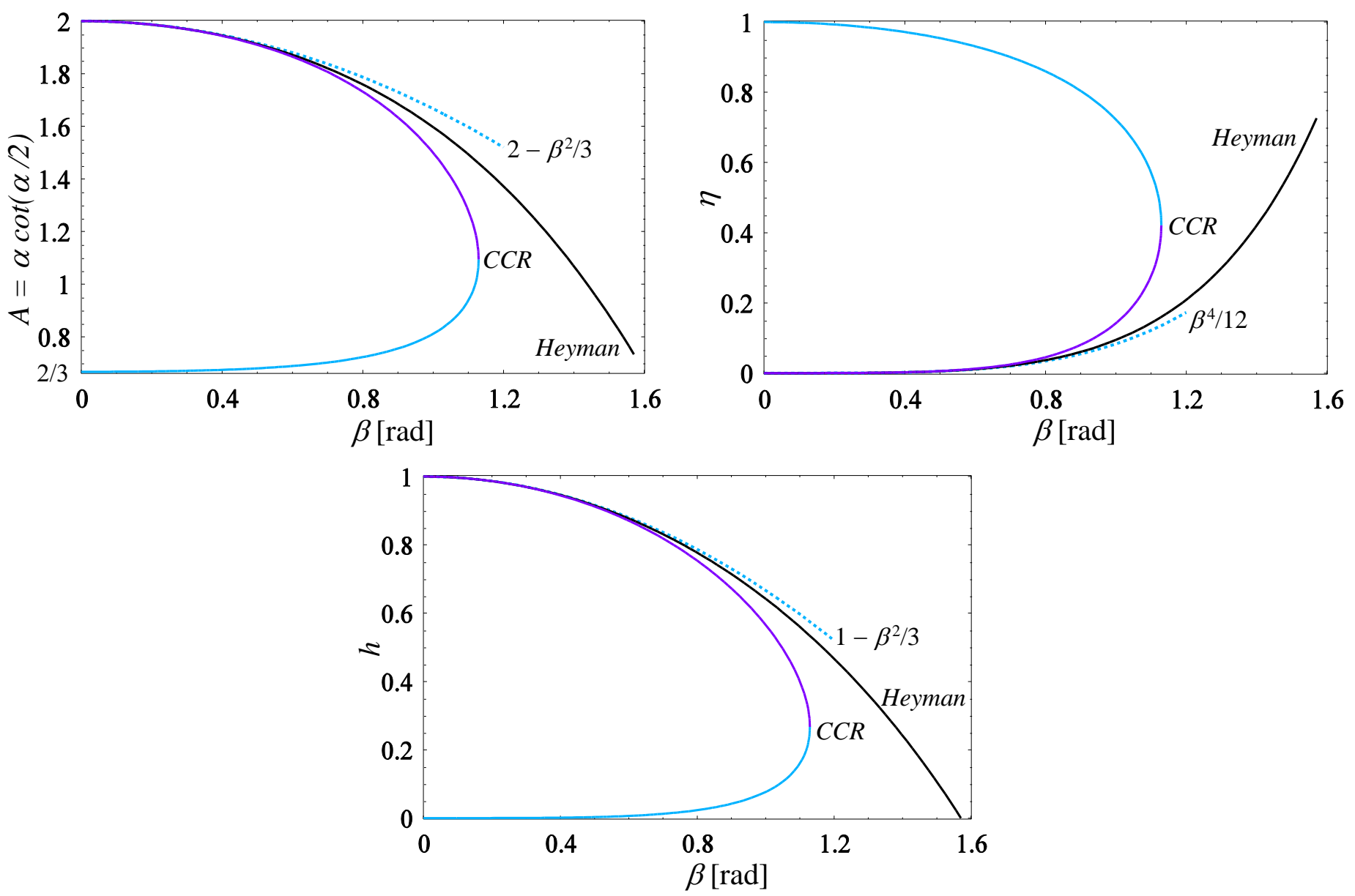

Figure 2: Analytical parametric plot of solution triplet $A(\beta), \eta(\beta), h(\beta)$ as a function of inner hinge angular position $\beta$ for CCR and Heyman solutions, with common trends for small $\beta(A=\alpha \cot (\alpha / 2), \alpha$ : half-angle of embrace; $\eta$ : thickness to radius ratio; $b$ : nondimensional horizontal thrust; refer to Fig. 1). 
This remarkably shows that $\eta(b)$ becomes a non-linear increasing function at decreasing $h$ and that $\beta(b)$, in the pre-peak branch, is also a non-linear increasing function at lowering $h$. Thus, if $h$ is set by external conditions (e.g., here, through friction reduction), in the least-thickness limit equilibrium state, $\eta$ and $\beta$ vary accordingly to the trends represented in Figs. 3a,b (non-linearly and with opposite concavities). These considerations display a crucial implication in the forthcoming investigation analysis at reducing friction.

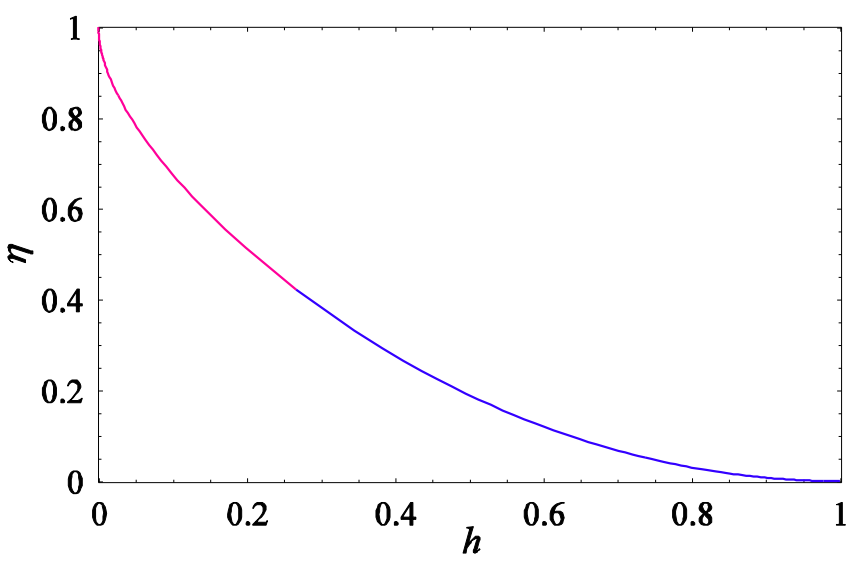

(a)

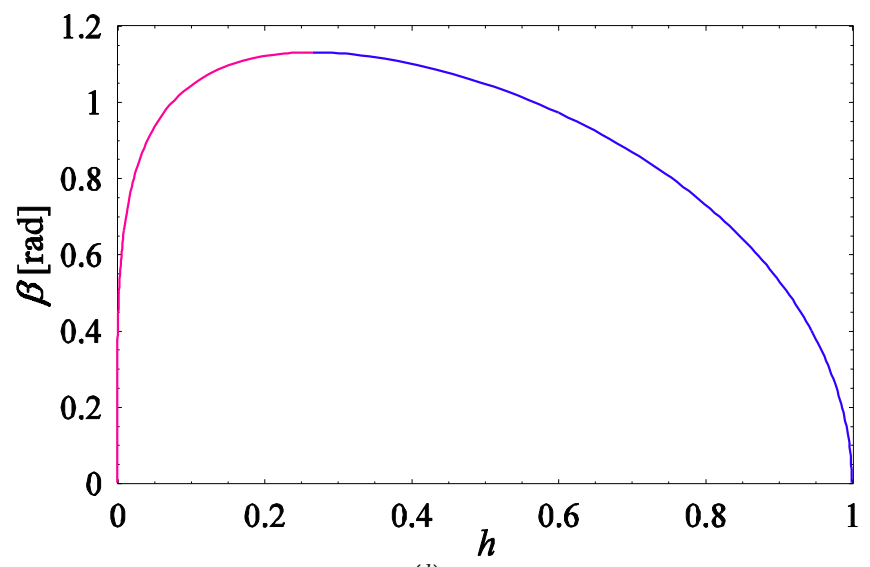

(b)

Figure 3: Analytical parametric plot as a function of non-dimensional horizontal thrust $h(\beta)$ : (a) least thickness to radius ratio $\eta(\beta)$; (b) inner hinge angular position $\beta$.

\section{Reducing friction}

The whole above solution holds true in Heyman sense for high values (infinite, in the limit) of friction coefficient $\mu=\tan \varphi$, apt to prevent sliding failure within the arch. By imaging now to decrease friction coefficient $\mu$ (not present in system (1)) from infinity or from such high values, one seeks when, and where in the arch, a first sliding joint may arise, for a critical value of $\mu=\mu_{r m}$ marking the transition between purely-rotational and mixed sliding-rotational modes. This should occur when limit sliding activation condition $T / N= \pm \mu$ is reached for the first time, where $T(\beta)$ and $N(\beta)$ are the shear (clock-wise positive) and normal (compression positive) components of the internal thrust force at each theoretical (radial) joint of the continuous arch.

From the translational equilibrium of any upper portion $\mathrm{AB}$ of the half-arch of general half-opening $\beta$, one gets, in non-dimensional terms:

$$
\left\{\begin{array}{l}
t(\beta)=\frac{T(\beta)}{w r}=b \sin \beta-\beta \cos \beta \\
n(\beta)=\frac{N(\beta)}{w r}=b \cos \beta+\beta \sin \beta
\end{array}\right.
$$

It may be noticed that non-dimensional internal force components $t(\beta)$ and $n(\beta)$ are just functions of geometrical angular position variable $\beta$, at a given value of horizontal thrust $h$ (which is constant along the arch). Specifically, thickness to radius ratio parameter $\eta$ does not intervene in Eqns. (8).

At the shoulders of the arch $(\beta=\alpha)$, internal action component ratio $t / n$ becomes:

$$
\frac{t}{n}(\beta=\alpha)=\frac{b \sin \alpha-\beta \cos \alpha}{b \cos \alpha+\alpha \sin \alpha}
$$

For the complete semi-circular arch, at the half-arch extremes (namely crown A and shoulder $\mathrm{C}$ ) one has, respectively: $\beta=0, t=0, n=h$, thus $t / n=0 ; \beta=\alpha=\pi / 2, t=h \sin \alpha-\alpha \cos \alpha=h, n=b \cos \alpha+\alpha \sin \alpha=\pi / 2$, thus $t / n=2 h / \pi$. 
Plots in Fig. 4a represent the variation of local slope $t / n$ of the internal thrust (to the local joint normal) at variable half-opening $\beta$ of upper portion $\mathrm{AB}$, for fixed values of $h$ ranging from 0.1 to 1 , together with the maps of stationary points appearing in the $t / n$ curves at variable $h$.

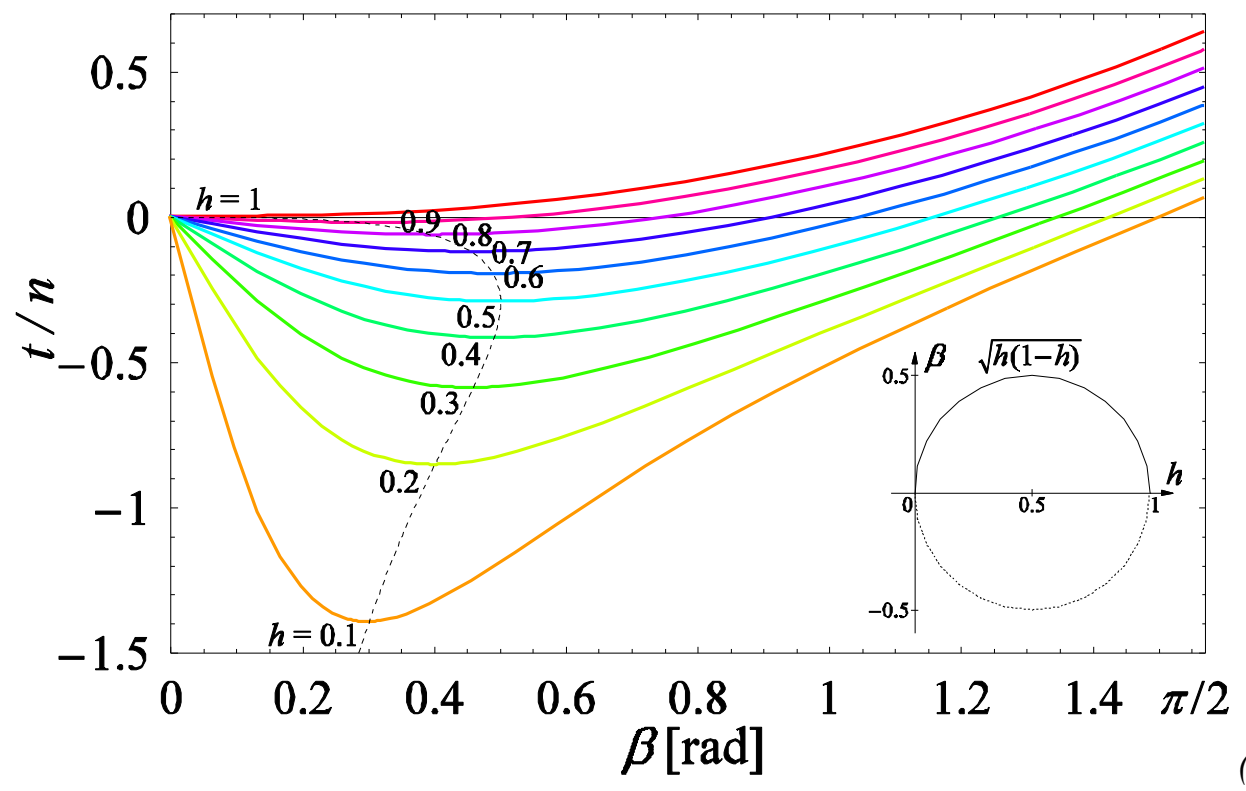

(a)

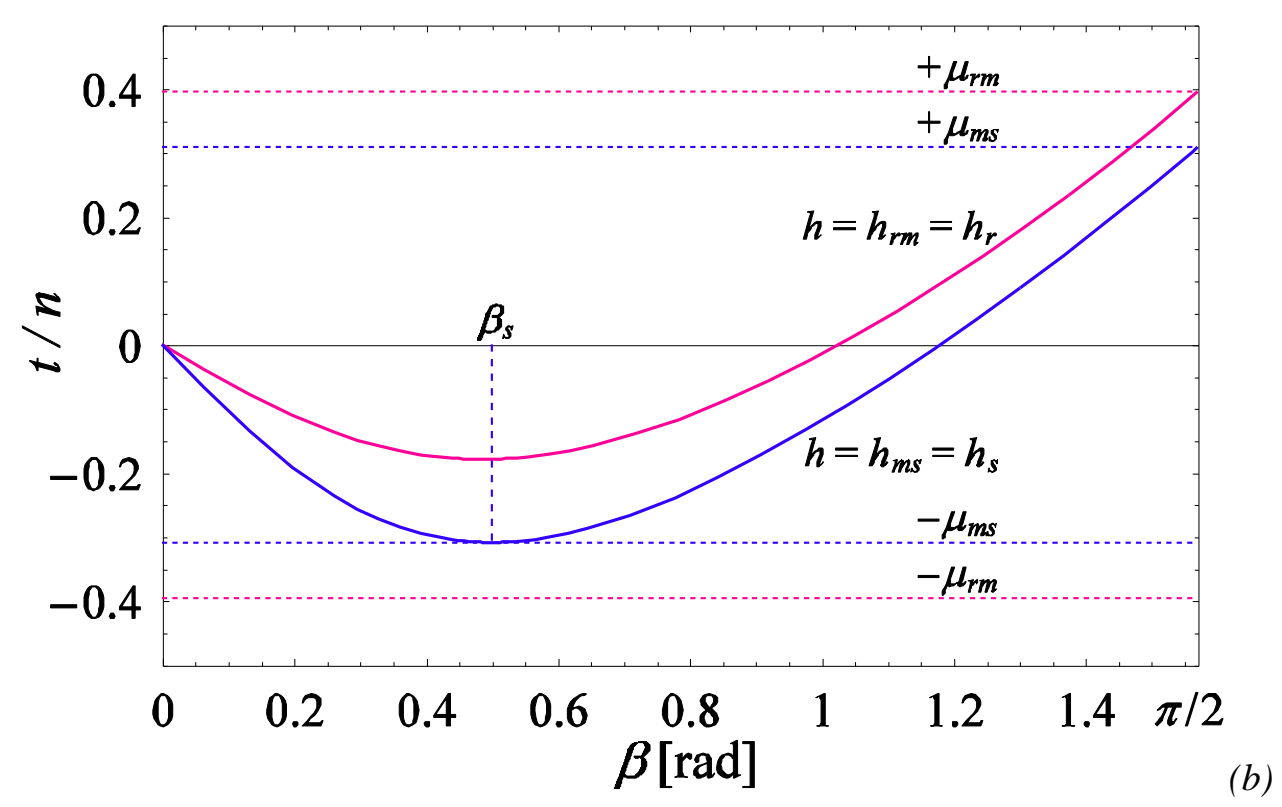

Figure 4: Local slope $t / n$ of non-dimensional shear/normal internal force components at variable $\beta$ for the complete semi-circular arch $(\alpha=\pi / 2)$ : (a) curves for different values of non-dimensional horizontal thrust $h$, with stationary maps; (b) limit situations of collapse mode transition with sliding activation at $\mu=\mu_{r m}$ and $\mu=\mu_{m s}$.

Two main facts clearly appear from the inspection of Fig. 4a: absolute maxima of thrust slope are always attained at the shoulder $(\beta=\alpha=\pi / 2)$; relative minima (maxima on negative side) occur at stationary points at intermediate locations $0 \leq \beta \leq 0.5$, at variable thrust $0 \leq b \leq 1$. This shows, on one hand, that the first sliding joint, at decreasing $\mu$, should appear at the shoulder when $t / n=+\mu$, leading to:

$$
h=h_{\mu}=\alpha \frac{\cos \alpha+\mu \sin \alpha}{\sin \alpha-\mu \cos \alpha}
$$


which provides transition mark $\mu=\mu_{r m}$ at $h=h_{r m}=h_{r}$ and then fixes the horizontal thrust within the arch, $b=h_{m}(\mu)$, under mixed sliding-rotational mode, as linearly decreasing with friction coefficient $\mu$ :

$$
\mu_{r m}=\frac{h_{r}}{\pi / 2}=\frac{2}{\pi} h_{r}=0.395832\left(\phi_{r m}=21.5952^{\circ}\right), h_{r m}=h_{r}=0.621772 ; h_{m}(\mu)=h_{\mu}(\mu)=\frac{\pi}{2} \mu
$$

Notice that generally $h_{\mu}(\mu)$ is a non-linear function of $\mu$, at variable $\alpha$. For the complete semi-circular arch $(\alpha=\pi / 2$; $\cos \alpha=0, \sin \alpha=1)$, it just becomes a linear function of $\mu$, namely $h_{\mu}=\pi / 2 \mu$.

On the other hand, in analytically seeking the stationary points marked in Fig. 4a, one then searches for the additional joint where further sliding may occur. Thereby, the corresponding stationary condition leads to:

$$
\frac{d}{d \beta}\left(\frac{t}{n}\right)=\left(\frac{t}{n}\right)^{\prime}=\frac{t^{\prime} n-t n^{\prime}}{n^{2}}=\frac{1}{n}\left(t^{\prime}-\frac{t}{n} n^{\prime}\right)=0 \Rightarrow \frac{t}{n}=\frac{t^{\prime}}{n^{\prime}}=\frac{-(1-b) \cos \beta+\beta \sin \beta}{(1-b) \sin \beta+\beta \cos \beta}
$$

Thus, since such sliding joint is activated when, there, $t / n=-\mu$, while $h=h_{\mu}$, one has to solve the following system of three governing equations (independently from thickness parameter $\eta$ ):

$$
\left\{\begin{array}{lll}
\frac{t}{n}=\frac{t^{\prime}}{n^{\prime}} & \Leftrightarrow b^{2}-b+\beta^{2}=0 \\
\frac{t}{n}=-\mu & \Leftrightarrow(\sin \beta+\mu \cos \beta) b-\beta(\cos \beta-\mu \sin \beta)=0 \\
b=b_{\mu}=\alpha \frac{\cos \alpha+\mu \sin \alpha}{\sin \alpha-\mu \cos \alpha} & \Leftrightarrow(\sin \alpha-\mu \cos \alpha) b-\alpha(\cos \alpha+\mu \sin \alpha)=0
\end{array}\right.
$$

Remark that Eqn. (13)a states the map of stationary points of $t / n$ as $\beta^{2}=h-b^{2}=h(1-b)$ or $\beta^{2}+(h-1 / 2)^{2}=(1 / 2)^{2}$ (see the circle insert in Fig. 4a). This is also obtained either by eliminating couple $(\alpha, \mu)$ from system (13), or variable $\mu$ from Eqns. (13) $)_{a}$ and (13) $b$.

Instead, by eliminating couple $(\mu, h)$ from system (13), and couple $(\alpha, h)$ from system (13) or variable $b$ from Eqns. (13) and (13) b, one also gets, respectively:

$$
\begin{aligned}
& {[2 \beta+\sin 2(\alpha+\beta)] \alpha^{2}-\left[1-4 \beta^{2}-\cos 2(\alpha+\beta)\right] \alpha+\beta^{2}[2 \beta-\sin 2(\alpha+\beta)]=0} \\
& (2 \beta+\sin 2 \beta) \mu^{2}-2 \cos 2 \beta \mu+2 \beta-\sin 2 \beta=0
\end{aligned}
$$

the latter equation leading to following symbolic solution $\mu=\mu^{ \pm}(\beta)$ :

$$
\mu^{ \pm}(\beta)=\frac{\cos 2 \beta \pm \sqrt{1-4 \beta^{2}}}{2 \beta+\sin 2 \beta}
$$

where higher root $\mu^{+}$holds true for $\mu \geq \cos 1 /(1+\sin 1)=0.293408$, with $\beta \leq 1 / 2$.

Thus, from Eqn. (14), with $\alpha=\pi / 2$, one may numerically solve for position $\beta=\beta_{s}$ of the new sliding joint. Then, in cascade, at that value of $\beta=\beta_{s}$, Eqn. (15), or Eqn. (16), leads to transition friction coefficient $\mu_{m s}$ (higher root); finally, Eqn. (13)a gives, at $\beta=\beta_{s}$, thrust $h_{m s}$ (lower root, corresponding to $b_{m s}=h_{\mu m s}$ ).

In doing so, or either by directly numerically solving system (13), one gets:

$$
\beta_{s}=0.499796 \mathrm{rad}=28.6362^{\circ}, \quad \mu_{m s}=0.309215\left(\phi_{m s}=17.1824^{\circ}\right), \quad h_{m s}=0.485714
$$


Notice that, once friction mark $\mu_{m s}$ is known, thus $h_{m s}=\pi / 2 \mu_{m s}$, stationary condition $(13)_{\mathrm{a}}$, i.e. $\beta^{2}=h(1-h)$, gives the following explicit expression of the angular position of the inner sliding joint for the purely-sliding collapse mode:

$$
\beta_{s}=\sqrt{h_{m s}\left(1-h_{m s}\right)}=\sqrt{\frac{\pi}{2} \mu_{m s}\left(1-\frac{\pi}{2} \mu_{m s}\right)}
$$

Since $h_{m s}$ is near $0.5, \beta_{s}$ is also near 0.5 (see circle insert in Fig. 4a).

\section{Mixed sliding-rotational mode}

At this stage, the friction boundaries for the appearance of the mixed sliding-rotational collapse mode have been located. Notice that:

- at $\mu=\mu_{r m}$, any 2-dof linear combination of 1-dof modes in Figs. 1b and 1c is possible;

- at $\mu=\mu_{m s}$, any 2-dof linear combination of 1-dof modes in Figs. 1c and 1d is feasible;

- in range $\mu_{m s}<\mu<\mu_{r m}$, the mixed-mode mode in Fig. 1c is found, with variable position $\beta_{m}(\mu)$ of inner hinge B, thickness to radius ratio $\eta_{m}(\boldsymbol{\mu})$ and horizontal thrust $h_{m}(\boldsymbol{\mu})$, at variable friction coefficient $\mu$.

This last occurrence is ruled by a new system of governing equations, in place of those in system (1), in which second equilibrium Eqn. (1) $\mathrm{b}$ is replaced by sliding equation $h=h_{\mu}=\pi / 2 \mu$, namely:

$$
\left\{\begin{array}{l}
b=b_{1}(\eta, \beta) \\
b=b_{\mu}(\alpha, \mu) \\
b=b_{e}(\eta, \beta)
\end{array}\right.
$$

The solution of this system actually brings back to the previous analysis for the purely-rotational mode. Indeed, equations $h=b_{1}$ and $h=h_{e}$ are still the same, with same solutions (5) and $(5)_{\mathrm{c}}$ for $\eta(\beta)$ and $h(\beta)$, as previously explained. By setting $h=h_{\mu}=\pi / 2 \mu$ in the expression of $h(\beta)$ in Eqn. (5) $)_{c}$ and solving for $\mu(\beta)=b(\beta) 2 / \pi$, or by eliminating $h=\pi / 2 \mu$ in two Eqns. (19) and (19) c, this leads to trends $\eta_{m}(\beta)$ and $\mu_{m}(\beta)$. These can be analytically plotted, by parametric plots at variable $\beta_{r m} \leq \beta \leq \beta_{m s}$ (Figs. 7-8, Section 4), where $\beta_{m s}$ can be found from $\mu(\beta)$, Eqn. (5)b, at $\mu=\mu_{m s}$. Similarly, $\eta_{m s}$ is found as $\eta\left(\beta_{m s}\right)$, at $h_{m s}=\pi / 2 \mu_{m s}$, so that:

$$
\beta_{m s}=1.05616 \mathrm{rad}=60.5134^{\circ}, \quad \eta_{m s}=0.200637, \quad h_{m s}=0.485714
$$

Since this leads to an increase of $\eta(\mu)$ at decreasing $\mu$, constant trace $\eta=\eta_{r}$ of the purely-rotational mode is abandoned, since $\eta(\mu)$ is higher and thus provides a new least thickness condition (Fig. 7). Accordingly, the hinge at the shoulder, co-present with the sliding joint there at $\mu=\mu_{r m}$, closes down. The inner haunch hinge B keeps instead on, and moves further down at decreasing $\mu$. Basically, the trends of $\eta(\mu)$ and $\beta(\mu)$ are read in Figs. 7 and 8 a, as they were in Figs. $3 \mathrm{a}$ and $3 \mathrm{~b}$ at decreasing $h$. Indeed, $h$ is limited by friction to $h_{\mu}=\pi / 2 \mu$, with the linear decreasing trend at lowering $\mu$ represented in Fig. 8b. Such a trend is linear for the exposed case of $\alpha=\pi / 2$.

Tab. 1 reports analytically-evaluated ("exact") mixed-mode collapse characteristics $\beta, \eta, h$ at variable friction coefficient $\mu$. At new transition $\mu=\mu_{m s}$, trends $\eta_{m}(\beta), \beta_{m}(\mu), h_{m}(\mu)$ stop. Limit equilibrium states associated to modes in Fig. $1 \mathrm{~d}$ do not depend on thickness parameter $\eta$, thus they would require any value of $\eta>\eta_{m s}$ in the least thickness condition. Thus, the inner hinge at the haunch also closes down and from the two modes in Figs. 1c and 1d, co-present at $\mu=\mu_{m s}$, only the purely-sliding mode in Fig. $1 \mathrm{~d}$ survives for $\eta>\eta_{m s}$. However, all these states at $\mu=\mu_{m s}$ are right-away limit equilibrium states, thus equilibrium is no-longer possible in practice, at any value $\eta>\eta_{m s}$.

Notice also that, at $\mu=\mu_{m s}$, inner hinge and sliding joints are differently located, respectively at $\beta=\beta_{m s}=1.05616 \mathrm{rad}=60.5134^{\circ}$ (hinge joint) and $\beta=\beta_{s}=0.499796 \mathrm{rad}=28.6362^{\circ}$ (sliding joint), thus interestingly at nearly $60^{\circ}$ and $30^{\circ}$.

The present analytical outcomes are going to be further commented in Section 4, with comparison as well to independent, matching, numerical results by a self-made spreadsheet implementation, as derived in the next section. 


\begin{tabular}{|c|c|c|c|c|c|c|c|c|c|}
\hline$\mu$ & $\eta$ & b & [rad] & [deg] & $\mu$ & $\eta$ & $b$ & [rad] & [deg] \\
\hline 0.7 & 0.107426 & 0.621772 & 0.951141 & 54.4963 & 0.35 & 0.152920 & 0.549779 & 1.01227 & 57.9986 \\
\hline 0.3959 & 0.107426 & 0.621772 & 0.951141 & 54.4963 & 0.34 & 0.163977 & 0.534071 & 1.02392 & 58.6661 \\
\hline 0.395832 & 0.107426 & 0.621772 & 0.951141 & 54.4963 & 0.33 & 0.175448 & 0.518363 & 1.03499 & 59.3003 \\
\hline 0.3958 & 0.107455 & 0.621721 & 0.951188 & 54.4991 & 0.32 & 0.187338 & 0.502655 & 1.04548 & 59.9016 \\
\hline 0.39 & 0.112750 & 0.612611 & 0.959654 & 54.9841 & 0.31 & 0.199653 & 0.486947 & 1.05540 & 60.4702 \\
\hline 0.38 & 0.122192 & 0.596903 & 0.973737 & 55.7910 & 0.3093 & 0.200531 & 0.485847 & 1.05608 & 60.5088 \\
\hline 0.37 & 0.132031 & 0.581195 & 0.987190 & 56.5618 & 0.309215 & 0.200637 & 0.485714 & 1.05616 & 60.5134 \\
\hline 0.36 & 0.142273 & 0.565487 & 1.00003 & 57.2974 & 0.3092 & \multicolumn{4}{|c|}{ No equilibrium solution } \\
\hline
\end{tabular}

Table 1: "Exact" critical solution values of triplet $\eta, h, \beta$ obtained for the complete semi-circular arch by the analytical analysis at variable friction coefficient $\mu$. Sliding joints appear at $\beta=\pi / 2 \operatorname{rad}=90^{\circ}$ for $0.309215=\mu_{m s} \leq \mu \leq \mu_{m m}=0.395832$ and at $\beta=\beta_{s}=0.499796 \mathrm{rad}=28.6362^{\circ}$ for $\mu=\mu_{m s}=0.309215$.

\section{Comment on present mixed mode at variable opening angle of the arch}

The previous relations, generally set for any half-angle of embrace $\alpha$, and illustrated in detail for the considered reference case of the complete semi-circular arch $(\alpha=\pi / 2)$, could be used to further explore the output of the discovered mixed mode, at reducing friction, for arches with variable opening angles. It can be shown that the analytical solution for the representation of the mixed mode here derived holds true for a range of opening angles up to the following limit one $\left(0<\alpha \leq \alpha_{l m}\right)$.

Indeed, if one seeks the condition leading to the common satisfaction of systems of three Eqns. (1) and three Eqns. (13), namely those that mark the rotational mode and the sliding mode, one achieves the following numerical solution of the six equations in six unknowns $\alpha_{l m}$ (limit opening angle for present mixed sliding-rotational mode), $\mu_{l m}$ (friction coefficient), $\eta_{l m}$ (thickness to radius ratio), $h_{l m}$ (non-dimensional horizontal thrust), $\beta^{\mathrm{R}_{l m}}$, (angular position of inner rotational joint), $\beta^{S_{l m}}$ (angular position of inner sliding joint):

$$
\begin{aligned}
& \alpha_{l m}=2.48716 \mathrm{rad}=142.504^{\circ}\left(A_{l m}=\alpha_{l m} \cot \left(\alpha_{l m} / 2\right)=0.844185\right), \\
& \mu_{l m}=1.41527\left(\varphi_{l m}=\operatorname{atan} \mu_{l m}=0.955669 \mathrm{rad}=54.7558^{\circ}\right), \\
& \eta_{l m}=0.679605, \quad h_{l m}=0.0978058, \\
& \beta_{l m}^{R}=1.03749 \mathrm{rad}=59.4435, \quad \beta_{l m}^{S}=0.297052 \mathrm{rad}=17.0198^{\circ}
\end{aligned}
$$

Thus, the documented solution of mixed mode is achieved until for an angle of embrace of about $\alpha_{l m}=142.5^{\circ}$, anyway requiring considerable friction (and thickness) for the arch to withstand under self-weight, in the limit condition of least thickness. At that value of $\alpha$ (marking a rather open, thick, horseshoe arch), the two solutions for rotational collapse and sliding collapse, together with that for mixed mode collapse, unify all together and are co-present at the same time for the values of the characteristic coefficients reported in Eqn. (21).

Further numerical results and representations of the collapse characteristics of the circular masonry arch at variable angle of embrace, implicitly described by the present analytical treatment, and fully consistent with such analytical solutions, are additionally presented in $[9,10]$, by a separate numerical treatment, based on a comprehensive non-linear Mathematical Programming formulation and implementation. A simpler, straightforward numerical strategy is instead proposed next, for independent and complete validation of the achieved analytical results. 


\section{NUMERICAL APPROACH}

A $\mathrm{n}$ immediate, self-implemented numerical algorithm for the individuation of the collapse modes of symmetric circular masonry arches has been further created within commercial spreadsheet software Excel, to independently inspect and validate the previous analytical outcomes on the arch's collapse characteristics, as revealed at reducing friction. It makes use of an optimisation function named "Solver", which allows for the selection of a GRG (Generalised Reduced Gradient) engine, towards the solution of smooth non-linear optimisation problems.

Similar, independent numerical tools of thrust-line or limit analysis, which may as well involve the use of spreadsheets and formulations of mathematical programming have been proposed [29-35]. An approach that shall be quite similar to the present one has been earlier developed by De Rosa and Galizia [34], for the analysis of pointed masonry arches. Discrete Element Method tools (see e.g. [8], and references therein quoted) may as well be employed toward the stated validation purpose, though the correct and precise evaluation of the threshold values of friction coefficients, and relevant arch thicknesses and collapse characteristics, with respect to the analytically determined benchmark values above, may constitute a rather delicate quest. The feeling, confirmed by first trials by an available DDA program already adopted in [8], within the present research endeavours, is that such numerical tools may not turn out as refined enough, to become capable to feel the subtle differences in the effects of variable friction, especially in correctly getting the transition values of friction coefficient, as exactly derived by the earlier analytical derivation. Likely, general trends may be qualitatively reproduced, in the best option, but it may be hard to recover true quantitative matchings with the analytical results. Thus, a separate and dedicated numerical tool was eventually conceived and implemented, as delivered in the present section, to provide a final confirmation of the analytical results, with truly matching outcomes, on a real quantitative basis, as then resumed in the subsequent section.

Input for the present spreadsheet numerical implementation within Excel is constituted by two kinds of data:

- geometrical: arch width $d$, mean radius $r$ (both nominally fixed to $1 \mathrm{~m}$ );

- material: limit tension stress $\sigma_{t}$ (set to zero, according to Heyman hypothesis 1), limit compression stress $\sigma_{c}$ (set to $1000 \mathrm{kN} / \mathrm{m}^{2}$, i.e. a high value apt to comply with Heyman hypothesis 2), variably-fixed friction coefficient $\mu$, weight per unit volume $\gamma\left(\right.$ set to $\left.25 \mathrm{kN} / \mathrm{m}^{3}\right)$.

Based on these input data, the trends of internal actions $N(\beta), T(\beta), M(\beta)$ along the arch are recovered by equilibrium and confronted to the limit values that define section resistance, specifically in terms of shear force $T$ and moment $M$. Then, an iterative procedure is put in place at variable thickness $t$, which constitutes the cell variable within the optimisation process, to evaluate the limit condition of least thickness. Characteristics $\beta$ (angular position of rupture joints), $\eta, b$ in the limit condition are then obtained, together with the variation of internal actions $N(\beta), T(\beta), M(\beta)$ along the arch, and associated thrust-line eccentricity $e(\beta)=M / N$ from geometrical centreline.

Thus, the numerical analysis is carried out by a static approach and the collapse mode is found out by the analysis in the limit thickness condition, as the output of the optimisation (thickness minimisation) process. Like that, notice that the collapse mode is not a priori imposed but truly obtained out of the numerical process.

The analysis is here carried out on a complete semi-circular masonry arch (angle of embrace $2 \alpha=\pi$ ). Due to symmetry, only one half of the arch is considered, with "hyperstatic" actions (moment $X=M_{A}$ and horizontal thrust $Y=H$ ) acting at geometrical centreline at crown section A. Statically-admissible configurations are those warranting equilibrium of any upper portion of the arch of angular opening $\beta$. They are described in non-dimensional terms by expressions (8), which determine shear $T(\beta)=t(\beta) w r$ and normal $N(\beta)=n(\beta) w r$ actions in each theoretical section of the arch, and by the following expression of moment $M(\beta)=m(\beta) w r^{2}$. Consistently with relations (8), in non-dimensional terms:

$$
m(\beta)=\frac{X}{w r^{2}}+h(1-\cos \beta)-(\beta \sin \beta-(1-\cos \beta))
$$

Cross section resistance may be set as follows. Given that $0=\sigma_{t} t d \leq N(\beta) \leq \sigma_{c} t d$ should always be satisfied within the arch, focus is made on moment and shear resistances. Since eccentricity $e(\beta)=M(\beta) / N(\beta)$, with $N(\beta)>0$ for $h>0$ (compression), should not exceed $\pm t / 2$ (no tensile strength) and assuming that shear is limited by a Coulomb friction law with friction coefficient $\mu$, one states the following two resistance inequalities to hold:

$$
|M(\beta)| \leq N(\beta) \frac{t}{2} ; \quad|T(\beta)| \leq \mu N(\beta)
$$


Within the optimisation process, toward the determination of least-thickness condition $t=t_{\min }$ still making the whole self-standing equilibrium possible, cell constraints are then represented by resistance conditions (23) and by the enforcement that, at crown section $A, Y=H>0$ and $|X|=\left|M_{A}\right| \leq Y t / 2$. Equilibrium values of $N(\beta), T(\beta), M(\beta)$ from Eqns. (8) and (22) are then determined at discretised angular positions, with angle-step $\Delta \beta=0.001 \mathrm{rad}$. The optimum search of $t_{\min }$ is iteratively performed, by varying the initial values of $X=M_{A}, Y=H$ and $t$, in order to satisfy all given constraints (with tolerances in the order of $10^{-6}$ ). Rotational and sliding joints are respectively detected, and their angular position $\beta$ recorded, when limit conditions (23) and (23) $)_{\mathrm{b}}$, with numerical equalities, are reached (Fig. 5).

A high value of friction coefficient is first set, namely $\mu=0.7\left(\varphi \cong 35^{\circ}\right)$, which should allow for predicting a purely-rotational collapse mode, due to Heyman hypothesis 3 (see analytical results in Tab. 1). The analysis is then repeated at lowering values of friction coefficient $\mu$, which has been reduced by steps up to $\Delta \mu=0.0001$, until the numerical algorithm becomes no-longer able to find equilibrium solutions. Transition conditions at $\mu=\mu_{r m}$ and $\mu=\mu_{m s}$ have been numerically found, by looking at activated hinge and/or sliding joints (Figs. 5 and 6). Results have been recorded in terms of characteristic arch parameters $\beta, \eta, h$ at variable $\mu$. Salient numerical outcomes are reported in Tab. 2 below (to be consistently compared with analytical results in earlier Tab. 1).

It may be noted from Tab. 2 that for $\mu=0.3959\left(\varphi=21.5986^{\circ}\right)$ the algorithm numerically detects the simultaneous presence of a rotational and a sliding joint at $\beta=\alpha=90^{\circ}$. This provides a consistent numerical estimate of "exact" friction coefficient $\mu_{r m}=0.395832\left(\varphi_{r m}=21.5952^{\circ}\right)$, as earlier analytically derived. Further, the lack of equilibrium solutions for $\mu<0.3093\left(\varphi<17.1868^{\circ}\right)$ provides a consistent numerical approximation of "exact" bound $\mu_{m s}=0.309215\left(\varphi_{m s}=17.1824^{\circ}\right)$.

These numerical results are also consistent with earlier independent numerical outcomes in [25-28], as mentioned in the Introduction. Also, the approximate numerical values of characteristic parameters $\beta, \eta, h$ fit quite well with the "exact" predictions from the analytical approach (Tab. 1), as analysed, represented and resumed next.

\begin{tabular}{|c|c|c|c|c|c|c|c|c|c|}
\hline$\mu$ & $\eta$ & $b$ & $\begin{array}{l}\text { Hinge } \\
\text { joints } \\
\beta[\mathrm{deg}]\end{array}$ & $\begin{array}{l}\text { Sliding } \\
\text { joints } \\
\beta \text { [deg] }\end{array}$ & $\mu$ & $\eta$ & $b$ & $\begin{array}{l}\text { Hinge } \\
\text { joints } \\
\beta \text { [deg] }\end{array}$ & $\begin{array}{l}\text { Sliding } \\
\text { joints } \\
\beta \text { [deg] }\end{array}$ \\
\hline 0.7 & 0.107426 & 0.621772 & $0-54.4883-90$ & - & 0.35 & 0.152920 & 0.549779 & $0-58.0120$ & 90 \\
\hline 0.396 & 0.107426 & 0.621772 & $0-54.4883-90$ & - & 0.34 & 0.163977 & 0.534071 & $0-58.5563$ & 90 \\
\hline 0.3959 & 0.107663 & 0.621878 & $0-54.4883-90$ & 90 & 0.33 & 0.175448 & 0.518363 & $0-59.3011$ & 90 \\
\hline 0.3958 & 0.107456 & 0.621721 & $0-54.4883$ & 90 & 0.32 & 0.187338 & 0.502655 & $0-59.9027$ & 90 \\
\hline 0.39 & 0.112750 & 0.612611 & $0-54.9753$ & 90 & 0.31 & 0.199653 & 0.486947 & $0-60.5043$ & 90 \\
\hline 0.38 & 0.122191 & 0.596903 & $0-55.7774$ & 90 & 0.3094 & 0.200406 & 0.486004 & $0-60.5043$ & 90 \\
\hline 0.37 & 0.132031 & 0.581195 & $0-56.6082$ & 90 & 0.3093 & 0.200531 & 0.485847 & $0-60.5043$ & 28.6479-90 \\
\hline 0.36 & 0.142273 & 0.565487 & $0-57.2958$ & 90 & 0.3092 & \multicolumn{4}{|c|}{ No equilibrium solutio } \\
\hline
\end{tabular}

Table 2: Approximate critical solution values of triplet $\eta, h, \beta$ obtained for the complete semi-circular arch by the numerical analysis at variable friction coefficient $\mu$.

\section{SUMMARY OF ANALYTICAL AND NUMERICAL OUTCOMES}

$\mathrm{F}$ igs. 7-8 below all together resume the present analytical and numerical results, by showing classical collapse characteristics of the masonry arch $\eta, \beta, b$ at variable (reducing) friction coefficient $\mu$. Numerical data out of the analysis in Section 3 are over-scored with cross markings on continuous analytical trends (parametric plots) out of the "exact" analysis in Section 2, with very good matching among them.

Specifically, Fig. 7 first reports a main outcome of the derivation, as the limit curve in the $(\eta, \mu)$ plane, in the sort of typical representation pointed out by Gilbert et al. [25] and Sinopoli et al. [26-28], and recently by Aita et al. [23], there generalised to masonry arches of various typologies and shapes. 

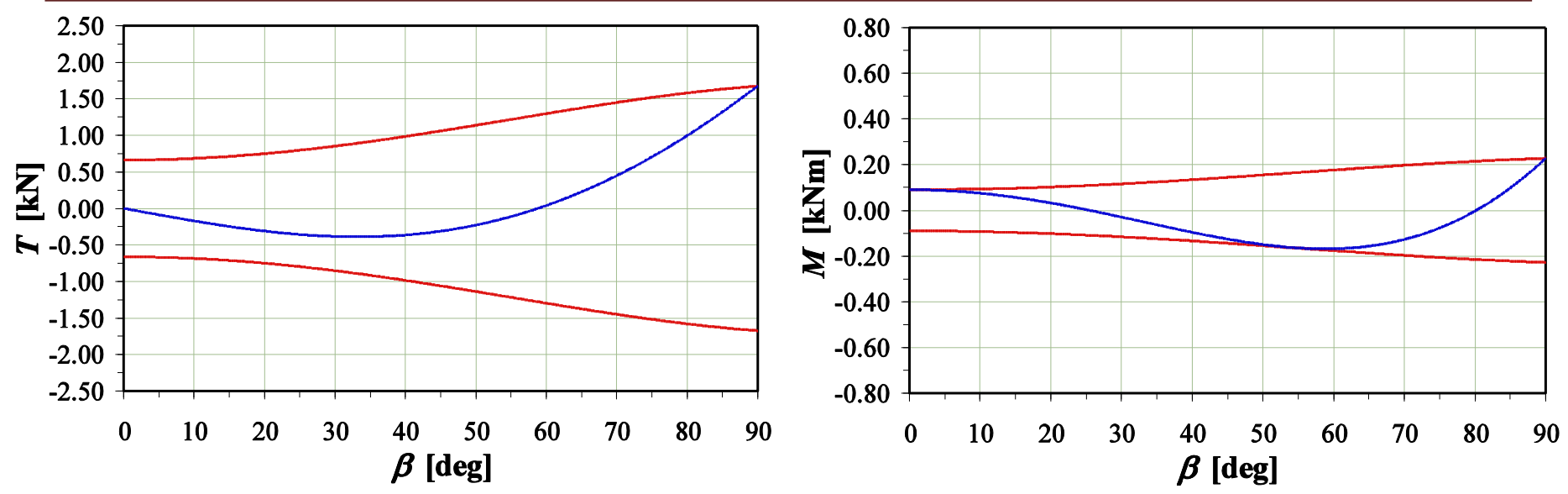

Figure 5: Numerical optimisation results for the trends of shear $T(\beta)$ and moment $M(\beta)$ at friction coefficient $\mu=0.3959$.
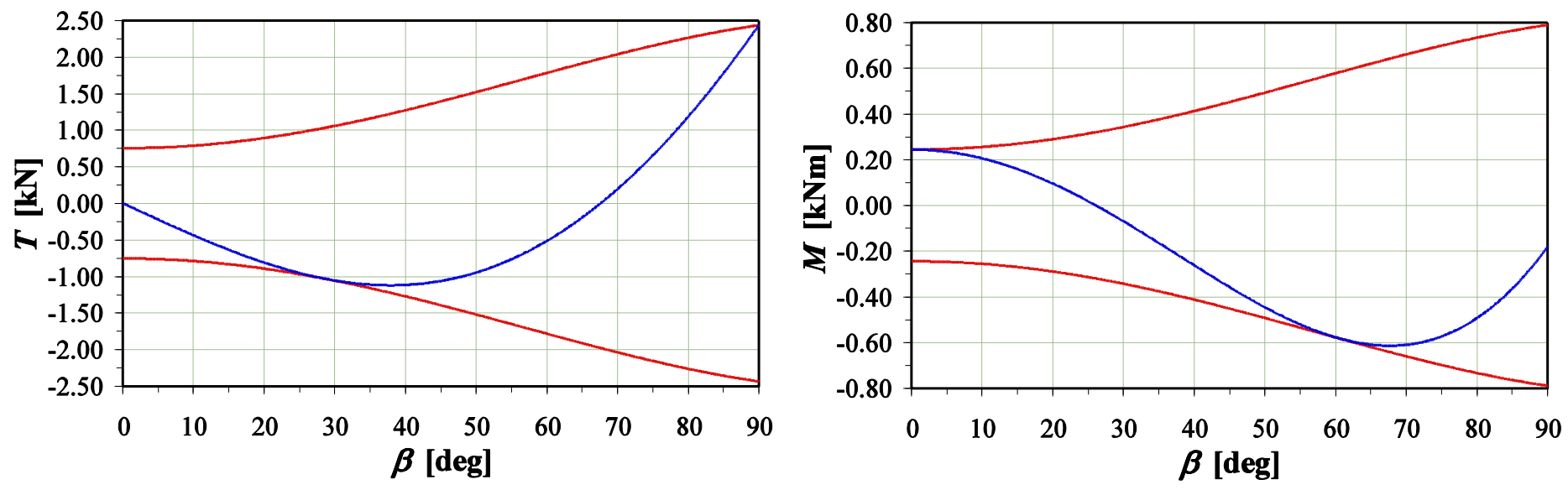

Figure 6: Numerical optimisation results for the trends of shear $T(\beta)$ and moment $M(\beta)$ at friction coefficient $\mu=0.3093$.

Moreover, Fig. 8 accordingly as well shows the non-linear trend of inner angular position $\beta$ variation at reducing friction $\mu$ in the mixed collapse mode and the associated (linear) decreasing trend of non-dimensional horizontal thrust $h$, as limited by reducing friction $\mu$ at the sliding joint forming at the springing of the arch at $\beta=\alpha=\pi / 2$. This constitutes a key feature for the appearance and interpretation of the arising mixed mode, as revealed by the present analytical and numerical investigation on the role of reducing friction.

On the hierarchy of the collapse modes at variable (decreasing) friction coefficient $\mu$, it may be resumed that for $\mu>\mu_{r m}$ the collapse mode in the least-thickness condition is purely-rotational (Fig. 1b). Collapse characteristics $\beta, \eta, b$ remain unvaried at changing $\mu$ : since purely-rotational collapse is uniquely determined by arch geometry, they are not dependent on the values of friction coefficient if greater than $\mu_{r m}$.

At transition $\mu=\mu_{r m}=0.395832\left(\varphi_{r m}=21.5952^{\circ}\right)$, a first sliding joint appears at the shoulder of the arch $(\beta=\alpha=\pi / 2)$, for a non-dimensional horizontal thrust $h=h_{r}$ matching $h=h_{\mu}(\mu)=\pi / 2 \mu$. This marks the transition from purely-rotational to mixed sliding-rotational collapse modes. The simultaneous presence of a hinge and a sliding joint at the shoulders is detected in both analytical and numerical analyses. The 2-dof collapse mode in such a transition state could be represented by any linear combination of 1-dof modes in Figs. 1b and 1c.

When friction coefficient $\mu$ is then further decreased from $\mu_{r m}$, non-dimensional horizontal thrust $h$ keeps fixed by friction as $h=h_{\mu}(\mu)$. The least thickness required for equilibrium is forced to increase. Indeed, since a lower friction coefficient is related to a lower resistance to sliding, a larger section (thickness) is needed to prevent collapse. Also, the purely-rotational collapse mode cannot be further triggered, since a thickness larger than value $\eta_{r}$ required by purely-rotational collapse (independent on $\mu$ ) is needed to avoid sliding. Hence, the collapse mode that appears first, when thickness is decreased from a super-critical value to the critical one, is the mixed sliding-rotational mode in Fig. 1c. At the same time, the inner 
hinge at the haunches moves further down, at decreasing $\mu$, from the location at purely-rotational collapse. This is due to the non-linear increasing trends of $\eta$ and $\beta$ at lowering $h$ (fixed here by friction) in the purely-rotational solution (Fig. 3).

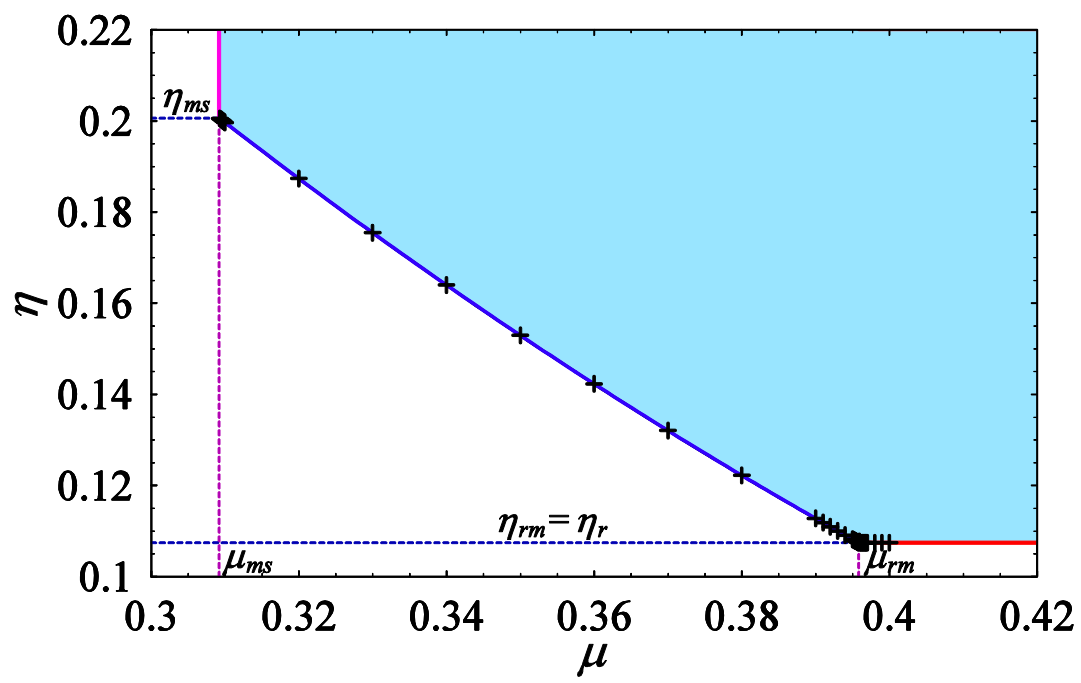

Figure 7: Least thickness to radius ratio $\eta$ at variable friction coefficient $\mu$, obtained by the analytical and numerical analyses. The mixed sliding-rotational mode range, $\mu_{m s}<\mu<\mu_{r m}$, is limited by $\mu_{m s}=0.309215\left(\varphi_{m s}=17.1824^{\circ}\right)$ and $\mu_{r m}=0.395832\left(\varphi_{r m}=21.5952^{\circ}\right)$. The shaded region represents the possible arch equilibrium states of couples $(\mu, \eta)$. Reducing friction $\mu$ sets a required increase of least thickness to radius ratio $\eta$.

At $\mu=\mu_{m s}=0.309215\left(\varphi_{m s}=17.1824^{\circ}\right)$ an additional sliding joint opens up at $\beta_{m s}=0.499796 \mathrm{rad}=28.6362^{\circ}$, when $\eta=\eta_{m s}=0.200637$, with $h=h_{m s}=0.485714$. At $\eta=\eta_{m s}$, this sliding joint coexists with a hinge at the haunches at $\beta_{m}\left(\mu_{m s}\right)=1.05616 \mathrm{rad}=60.5134^{\circ}$, and the corresponding 2-dof mixed collapse mode would be any linear combination of 1-dof collapse modes in Figs. 1c and 1d. Any larger value of $\eta>\eta_{m s}$ would instead represent limit equilibrium conditions at $\mu=\mu_{m s}$ for which purely-sliding collapse would develop (Fig. 1d). In practice, at $\mu=\mu_{m s}$ any value of $\eta>\eta_{m s}$ would correspond to limit states associated to purely-sliding modes in Fig. 1d and the arch would no longer be able to withstand under self-weight.

Nothing should be said, by the present static approach, for values of $\mu<\mu_{m s}$, since then equilibrium is no-longer possible at any value of $\eta$. Thus, it may be concluded that the shaded region in Fig. 7 represents the equilibrium states of couples $(\mu, \eta)$ allowing for arch equilibrium under self-weight. The inferior boundary of this domain is set by constant line $\eta=\eta_{r}$ at $\mu \geq \mu_{r m}$, then by curve $\eta=\eta_{m}(\mu)$ at $\mu_{m s} \leq \mu \leq \mu_{r m}$, finally by vertical line $\eta \geq \eta_{m s}$ at $\mu=\mu_{m s}$ (Fig. 7).

Tab. 3 below further resumes all the main solution characteristics obtained by the analytical results, for the various traced collapse modes of the complete semi-circular arch $(2 \alpha=\pi)$, at reducing friction, according to the above detailed discussion and the corresponding illustration of the collapse modes. Notice that the collapse mechanisms were already drawn, on scale, in Figs. $1 \mathrm{~b}, \mathrm{c}, \mathrm{d}$, as indeed corresponding to the main cases on the $1^{\text {st }} 3^{\text {rd }}$ and $5^{\text {th }}$ rows of Tab. 3, now reported. Remark that these constitute the three basic collapse cases that are revealed by the study. The other two intermediate rows in the table represent transition cases, among couples of them, where any possible combination of the two underlying mechanisms meeting at such a transition instance becomes possible.

\section{CONCLUSIONS}

$\mathrm{T}$ he role that friction coefficient $\mu$ at the theoretical (radial) joints of a continuous circular masonry arch plays in the definition of geometrical collapse of continuous arches has been fully investigated, with specific reference illustration to the classical case of the complete semi-circular arch (half-angle of embrace $\alpha=\pi / 2=90^{\circ}$ ). The analytical treatment presented in $[5,6,8]$ for purely-rotational collapse has been complemented and extended, by releasing Heyman hypothesis 3 of no sliding failure and looking at the induced changes of the collapse mode through the role of a reducing friction, leading to sliding onset. 

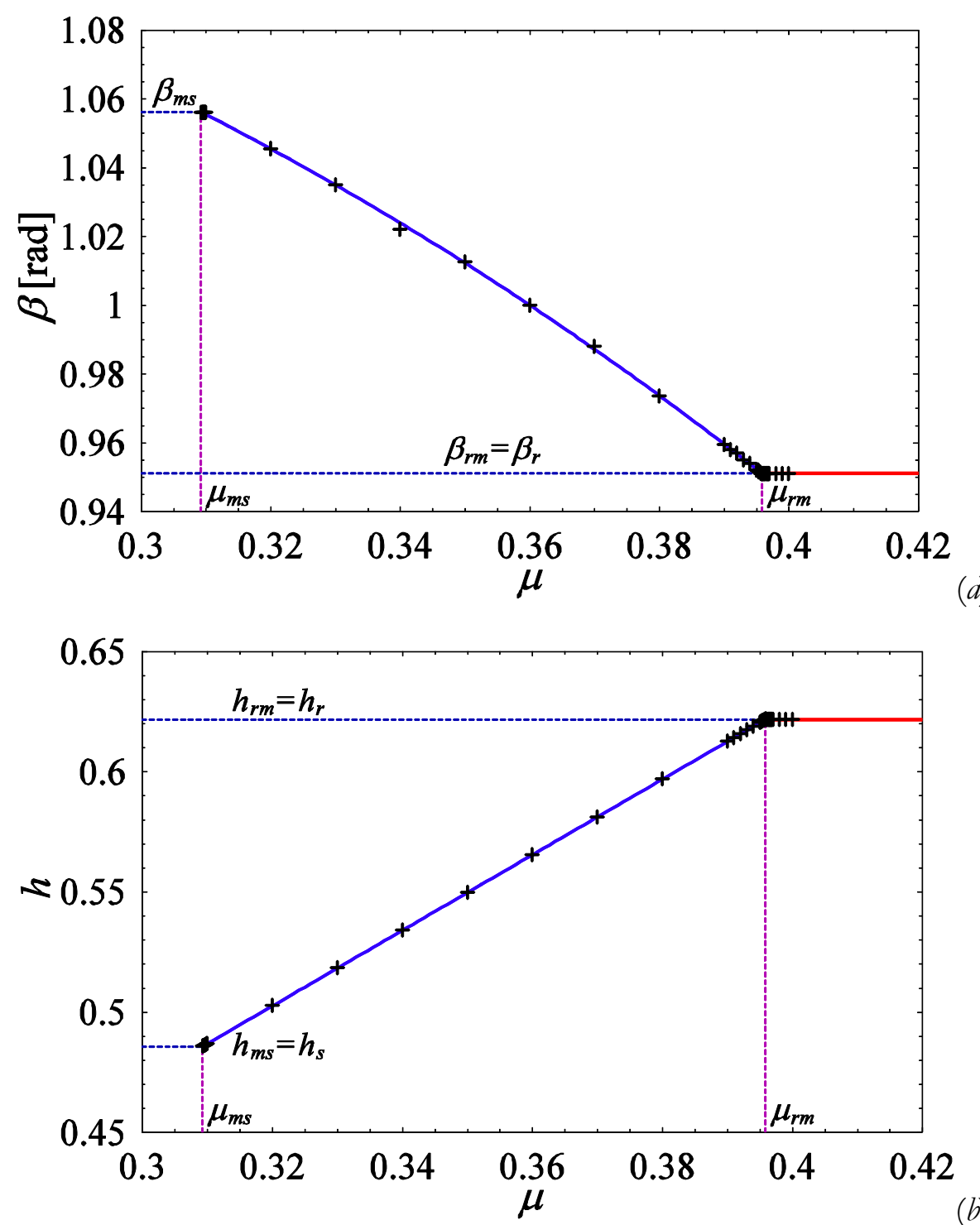

Figure 8: Trends of characteristic parameters $\beta, b$ at variable friction coefficient $\mu$, obtained by the analytical and numerical analyses: (a) inner hinge angular position $\beta$; (b) non-dimensional horizontal thrust $h$. Reducing friction $\mu$ sets a non-linear increase of angular position $\beta$ of the inner hinge and a linear (for $2 \alpha=\pi$ ) decrease of non-dimensional horizontal thrust $h$.

Then, at decreasing friction coefficient $\mu$, different masonry arch collapse modes have been located as follows (Figs. 1b-d, Tab. 3):

- for $\mu>\mu_{r m}$ classical purely-rotational collapse (Fig. 1b), with five hinges in the symmetric configuration of the whole arch (one at the crown, two at the haunches and two at the shoulders);

- for $\mu_{m s}<\mu<\mu_{r m}$ mixed sliding-rotational collapse (Fig. 1c), with three hinges in the whole arch, one at the crown and two at the haunches, and with two sliding joints at the shoulders;

- for $\mu=\mu_{m s}$ and $\eta>\eta_{m s}$ purely-sliding collapse (Fig. 1d), with four sliding joints in the whole arch, symmetrically placed at the shoulders and at the haunches (at an angle $\beta_{s} \simeq 30^{\circ}$ differing from that $\beta_{m}\left(\mu_{m s}\right) \simeq 60^{\circ}$ locating the last inner hinge position in the previous collapse mode at $\mu=\mu_{m s}$ ).

At two transition instances $\mu=\mu_{r m}$ and $\mu=\mu_{m s}$, 2-dof modes obtained as any linear combinations of the two adjacent 1 -dof modes above become possible.

The reducing friction range in which the newly (analytically) discovered mixed sliding-rotational mode occurs is quite narrow, with friction angles between near $22^{\circ}$ and $17^{\circ}$. This result obviously holds true for the ideal case of perfectly holding shoulders (i.e. no abutment settlements). As a crucial feature, at decreasing friction coefficient $\mu$, horizontal 
thrust $b$ decreases with it, as set by friction, linearly for $2 \alpha=\pi$, by ranging from $h_{r}$ to about $0.78 h_{r}$. At the same time, $\eta$ non-linearly increases from $\eta_{r}$ to about $1.87 \eta_{r}$ (then, critical thickness almost doubles) and $\beta$ also increases from near $\beta_{r} \simeq 54.5^{\circ}$ to about $1.11 \beta_{r} \simeq 60.5^{\circ}$. An inner sliding joint finally appears at $\mu=\mu_{m s}$ at a different $\beta_{s}$ location near $30^{\circ}$ from the crown.

\begin{tabular}{|c|c|c|c|}
\hline $\begin{array}{c}\text { Collapse } \\
\text { mode }\end{array}$ & $\begin{array}{l}\text { Friction } \\
\text { coefficient } \mu\end{array}$ & \multicolumn{2}{|c|}{$\begin{array}{l}\text { Collapse characteristics } \\
\beta, \eta, b(\alpha=\pi / 2)\end{array}$} \\
\hline $\begin{array}{l}\text { Purely- } \\
\text { rotational } \\
\qquad(r)\end{array}$ & $\mu>\mu_{r m}$ & $\left\{\begin{array}{l}b=b_{1}(\eta, \beta) \\
b=h_{e}(\eta, \beta)\end{array}+b=h_{2}(\eta, A(\alpha)) \Rightarrow\right.$ & $\left\{\begin{array}{l}\beta_{r}^{R}=0, \quad \beta_{r}^{R}=\pi / 2 \mathrm{rad}=90^{\circ} \\
\beta_{r}^{\mathrm{R}}=0.951141 \mathrm{rad}=54.4963^{\circ} \\
\eta_{r}=0.107426 \\
h_{r}=0.621772\end{array}\right.$ \\
\hline $\begin{array}{l}\text { Rotational/ } \\
\text { mixed shift } \\
\qquad(\mathrm{rm})\end{array}$ & $\begin{array}{c}\mu=\mu_{r m}=0.395832 \\
\left(\varphi_{r m}=21.5952^{\circ}\right)\end{array}$ & \multicolumn{2}{|c|}{$\begin{array}{l}\beta_{r m}^{R}=0 \\
\beta_{r m}^{R}=0.951141 \mathrm{rad}=54.4963^{\circ}, \quad \eta_{r m}=0.107426 \\
\beta_{r m}^{R}=\beta_{r m}^{S}=\pi / 2 \mathrm{rad}=90^{\circ}\end{array}$} \\
\hline $\begin{array}{l}\text { Mixed sliding } \\
\text { rotational } \\
\qquad(m)\end{array}$ & $\mu_{m s}<\mu<\mu_{r m}$ & $\left\{\begin{array}{l}b=h_{1}(\eta, \beta) \\
h=h_{e}(\eta, \beta)\end{array}+b=h_{\mu}(\alpha, \mu)\right.$ & $\left\{\begin{array}{l}\beta_{m}^{\mathrm{R}}=0, \quad \beta_{m}^{S}=\pi / 2 \mathrm{rad}=90^{\circ} \\
\beta_{m}^{\mathrm{R}}=\beta(\mu) \\
\eta_{m}=\eta(\mu) \\
b_{m}=b(\mu)=\pi / 2 \mu\end{array}\right.$ \\
\hline $\begin{array}{l}\text { Mixed/ } \\
\text { sliding shift } \\
\quad(m s)\end{array}$ & $\begin{array}{c}\mu=\mu_{m s}=0.309215 \\
\left(\varphi_{m s}=17.1824^{\circ}\right)\end{array}$ & \multicolumn{2}{|c|}{$\begin{array}{l}\beta_{m s}^{R}=0, \quad \beta_{m s}^{s}=\pi / 2 \mathrm{rad}=90^{\circ} \\
\beta_{m s}^{R}=1.05616 \mathrm{rad}=60.5134^{\circ}, \quad \eta_{m s}=0.200637, \quad h_{m s}=0.485714 \\
\beta_{m s}^{s}=0.499796 \mathrm{rad}=28.6362^{\circ}\end{array}$} \\
\hline $\begin{array}{l}\text { Purely- } \\
\text { sliding } \\
(s)\end{array}$ & $\mu=\mu_{m s}$ & \multicolumn{2}{|c|}{$\begin{array}{l}\beta_{s}^{S}=0.499796 \mathrm{rad}=28.6362^{\circ} \\
\beta_{s}^{S}=\pi / 2 \mathrm{rad} \quad=90^{\circ}\end{array}, \quad \eta_{s}>\eta_{m s}=0.200637, \quad h_{s}=h_{m s}=0.485714$} \\
\hline- & $\mu<\mu_{m s}$ & \multicolumn{2}{|c|}{ No equilibrium solution } \\
\hline
\end{tabular}

Table 3: Summary of analytical collapse characteristics at variable friction coefficient $\mu$ for the complete semi-circular arch with $2 \alpha=\pi$ (refer also to Fig. 1).

This analytical and numerical investigation has enquired the role of friction in the framework of classical Heyman masonry arch analysis. Focus has been explicitly made on the case of a complete semi-circular arch $(2 \alpha=\pi)$, to report detailed results and understand crucial features, specifically for the present mixed sliding-rotational mode. The analysis could be further generalised to cases of general half-opening angles $\alpha$, as implicitly analytically here defined and independently numerically outlined in $[9,10]$ by an innovative non-linear mathematical programming procedure, accounting for both static and kinematic admissibilities, all together. This considers a general formulation apt to address possible issues of non-normality in the prediction of the Limit Analysis formulation and potential instances of non-uniqueness in the determination of the limit thickness condition. Indeed, these aspects have been previously discussed in the literature [36-39], particularly in the context of accounting for friction effects in masonry constructions [40-45]. Specifically, Gilbert et al. [25] have mentioned that "Casapulla and Lauro (2000) bave identified a special class of non-associative friction problems for which provably unique solutions exist. The class comprises arches with symmetrical loading and geometry.", as handled in the present case. These outcomes have also been confirmed by the recent analysis by Aita et al. [23]. Despite, further, general analytical and numerical formulations shall investigate the subject, in inspecting if friction reduction effect may induce a resulting non-uniqueness in the prediction of the least-thickness condition, as instead still recorded in the present setting devoted to the analysis of symmetric masonry arches under self-weight, for more unspecific configurations and loading conditions. 
Further remarks on the present study, its implications, practical value and perspectives may be outlined as follows:

- Issue of dilatancy at the blocks' interfaces. Matter of dilatancy may be pertinent, like as due to interlocking arising among masonry joints, for the mutual surfaces of the chunks being not perfectly planar, with a relative roughness that shall locally be present there. Then, a little relative normal displacement among the joints may accompany a tangential relative displacement, when sliding is activated, the resulting friction angle being the arctangent of the relevant normal to sliding displacement ratio. This shall indeed introduce a further characteristic parameter of the joint, within the analysis, beyond the already considered main one, namely the friction coefficient. This further effect may likely be secondary in the analysis, explicitly focusing on the role of friction, in the considered context of masonry arches; a main novelty, in analytical terms. Possibly, the dilatancy effect may induce some variations in the threshold friction coefficient values that are derived within the present paper, for the potential sliding onset and the transitions among the various possible collapse modes, and for the recorded collapse characteristics. Moreover, it looks hard to state, a priori, if such a dilatancy effect may lay on the safe or the unsafe approximation side, for the critical arch behaviour. As a conjecture, the dilatancy process may lead to further resources of the arch to withstand under self-weight, if confinement within the arch shall hold true (like with firm supports). In that sense, the present analysis neglecting dilatancy may turn out to lay on the conservative side, meaning that the predicted least-thickness condition may anyway be safe (even a lower arch thickness may suffice for arch equilibrium, due to dilatancy). Certainly, dilatancy shall anyhow lead to another, different source of non-normality, and possible related effects. Thus, the perspective handling of the issue of dilatancy may certainly be appropriate, for a complete understanding of arch collapse but shall require a dedicated and comprehensive treatment, and may then constitute the subject of future research work.

- Issue of real friction values, in practical contexts, as pertinent to a reducing friction, apt to prevent/induce sliding. The present investigation is mainly and firstly endowed of a theoretical value. The results look useful in terms of the overall behaviour of the considered mechanical system; a full recognition of all its possible features. However, there may be reasons that may induce or be associated to a reducing friction effect among the blocks, as possibly connected to external conditions, like loosening of the joints, fading or spreading supports, percolation of rain, humidity, rubbish and mud within the joints, loosening of the mortar, if present, in cemented joints, and possible damage within that, existing interfaces between different composing materials (concrete, soil, earth, stone, masonry, etc.) among the arch's blocks, bearing interface on underlying pier, wall or ground with underfilled loosened material, like earth, natural sediments, etc. These effects may lead the friction coefficient values to reduce and possibly approach the critical ranges where sliding may be triggered, so that a verification about that shall anyway be attempted. Moreover, arches of different opening angles or of varied morphological shapes, symmetric or unsymmetric, do display a variation of the critical friction coefficient value possibly leading to the onset of sliding. Indeed, the discussion in Section 2.3, still concerning symmetric arches but with variable opening angles, already shows that the transition value of friction apt to prevent any sliding within the arch raises up to a value of friction coefficient around 1.4 (friction angle about $55^{\circ}$ ), for the limit case where the range of mixed mode vanishes (limit horseshoe arch). For this case, and other overcomplete arch cases, the critical value of friction leading to possible sliding onset may be nearing the range for practical applications, where, with the further possible intervention of some of the above effects, may really come to induce arch collapse with sliding. Thus, the issue of quantifying the amount of friction that shall be necessary to avoid sliding seems anyway much important, also in practical terms, for investigating the whole equilibrium ranges of the arch.

- Arch discretisation. The analysis actually refers to that of a continuous arch, i.e. in strict Heyman sense, where rupture joint may appear at critical locations, precisely where they shall be. This leads to the definition of the true least-thickness critical condition that nature by the gravitational field will find to let the arch to first collapse once thickness is gradually reduced. If fracture joints shall instead occur only at pre-defined locations, due to specific block arrangements, this should lead to lower critical thicknesses, for the arch to withstand. This aspect was widely discussed in previous works $[6,8]$, in the context of infinite friction, and shall apply as well in the present, finite friction one. Thus, specific block patterns, within the arch, may lead to some variations, of the general characteristics of a continuous arch, though the latter truly mark the real underlying least-thickness condition, at variable friction.

\section{ACKNOWLEDGEMENTS}

7 his work has been carried-out at the University of Bergamo, School of Engineering (Dalmine). The financial support by ministerial (MIUR) funding "Fondi di Ricerca d'Ateneo ex 60\%" at the University of Bergamo is gratefully acknowledged. 


\section{REFERENCES}

[1] Heyman, J. (1969). The safety of masonry arches, International Journal of Mechanical Sciences, 11(4), pp. 363-385, DOI: 10.1016/0020-7403(69)90070-8.

[2] Heyman, J. (1977). Equilibrium of Shell Structures, Clarendon Press, Oxford.

[3] Heyman, J. (1982). The Masonry Arch, Ellis Horwood Ltd., Chichester.

[4] Heyman, J. (2009). La coupe des pierres, In: Proceedings of the $3^{\text {rd }}$ International Congress on Construction History, Brandenburg University of Technology, Cottbus, Germany, 20-24 May 2009, 2, pp. 807-812.

[5] Rizzi, E., Cocchetti, G., Colasante, G., Rusconi, F. (2010). Analytical and numerical analysis on the collapse mode of circular masonry arches, In: Proceedings of $7^{\text {th }}$ International Conference on Structural Analysis of Historical Constructions (SAHC-2010), Eds. Xianglin Gu and Xiaobin Song, Shanghai, China, October 6-8, 2010, Trans Tech Publications, Switzerland, Periodical of Advanced Materials Research 133-134, pp. 467-472, DOI: 10.4028/www.scientific.net/AMR.133-134.467.

[6] Cocchetti, G., Colasante, G., Rizzi, E. (2011). On the analysis of minimum thickness in circular masonry arches. Part I: State of the art and Heyman's solution. Part II: Present CCR solution. Part III: Milankovitch-type solution, Applied Mechanics Reviews, ASME, September 01, 2011, 64(5), Paper 050802 (Oct. 01, 2012), pp. 1-27. DOI: $10.1115 / 1.4007417$.

[7] Rizzi, E., Colasante, G., Frigerio, A., Cocchetti, G. (2012). On the mixed collapse mechanism of semi-circular masonry arches, In: Proceedings of $8^{\text {th }}$ International Conference on Structural Analysis of Historical Constructions (SAHC-2012), Wroclaw, Poland, October 15-17, 2012, Ed. Jerzy Jasienko, DWE, Vol. 1, pp. 541-549.

[8] Rizzi, E., Rusconi, F., Cocchetti, G. (2014). Analytical and numerical DDA analysis on the collapse mode of circular masonry arches, Engineering Structures, 60(February 2014), pp. 241-257, DOI: 10.1016/j.engstruct.2013.12.023.

[9] Cocchetti, G., Rizzi, E. (2018). Limit analysis of circular masonry arches at reducing friction, In: Proceedings of the 10 ${ }^{\text {th }}$ International Masonry Conference (10 th $\left.I M C\right)$, Politecnico di Milano, July 9-11, 2018, Eds. Gabriele Milani, Alberto Taliercio and Stephen Garrity, The International Masonry Society (IMS), pp. 486-503.

[10] Cocchetti, G., Rizzi, E. (2019). Non-linear programming numerical formulation to acquire limit self-standing conditions of circular masonry arches accounting for limited friction, International Journal of Masonry Research and Innovation, Accepted 29 October 2019, in press (corrected proofs).

[11] Ochsendorf, J. (2002). Collapse of Masonry Structures, Doctoral Dissertation, University of Cambridge, UK.

[12] Ochsendorf, J. (2006). The masonry arch on spreading supports, The Structural Engineer, 84(2), pp. 29-36.

[13] Milankovitch, M. (1907). Theorie der Druckkurven, Zeitschrift für Mathematik und Physik, 55, pp. 1-27.

[14] Foce, F. (2007). Milankovitch's Theorie der Druckkurven: Good mechanics for masonry architecture, Nexus Network Journal, 9(2), pp. 185-210.

[15] Alexakis, H., Makris, N. (2013). Minimum thickness of elliptical masonry arches, Acta Mechanica, 224(12), pp. 2977-2991.

[16] Makris, N., Alexakis, H. (2013). The effect of stereotomy on the shape of the thrust-line and the minimum thickness of semicircular masonry arches, Archive of Applied Mechanics, 83(10), pp. 1511-1533.

[17] Alexakis, H., Makris, N. (2015). Limit equilibrium analysis of masonry arches, Archive of Applied Mechanics, 85(9-10), pp. 1363-1381.

[18] Bagi, K. (2014). When Heyman's Safe Theorem of rigid block systems fails: Non-Heymanian collapse modes of masonry structures, International Journal of Solids and Structures, 51(14), pp. 2696-2705.

[19] Lengyel, G. (2018). Minimum thickness of the gothic arch, Archive of Applied Mechanics, 88(5), pp. 769-788. DOI: $10.1007 / \mathrm{s} 00419-018-1341-6$.

[20] Cavalagli, N., Gusella, V., Severini, L. (2016). Lateral loads carrying capacity and minimum thickness of circular and pointed masonry arches, International Journal of Mechanical Sciences, 115-116(September 2016), pp. 645-656.

[21] Nikolić, D. (2017). Thrust line analysis and the minimum thickness of pointed masonry arches, Acta Mechanica, 228(6), pp. 2219-2236. DOI: 10.1007/s00707-017-1823-6.

[22] Gáspár, O., Sipos, A.A., Sajtos, I. (2018). Effect of stereotomy on the lower bound value of minimum thickness of semi-circular masonry arches, International Journal of Architectural Heritage, 12(6), pp. 899-921. DOI: $10.1080 / 15583058.2017 .1422572$.

[23] Aita, D., Barsotti, R., Bennati, S. (2019). Looking at the collapse modes of circular and pointed masonry arches through the lens of Durand-Claye's stability area method, Archive of Applied Mechanics, pp. 1-18.

DOI: 10.1007/s00419-019-01526-z. 
[24] Angelillo, M. (2019). The model of Heyman and the statical and kinematical problems for masonry structures, International Journal of Masonry Research and Innovation, 4(1-2), pp. 14-31. DOI: 10.1504/IJMRI.2019.096820.

[25] Gilbert, M., Casapulla, C., Ahmed, H.M. (2006). Limit analysis of masonry block structures with non-associative frictional joints using linear programming, Computers and Structures, 84(13-14): pp. 873-887.

[26] Sinopoli, A., Corradi, M., Foce, F. (1997). Modern formulation for preelastic theories on masonry arches, Journal of Engineering Mechanics, ASCE, 123(3): pp. 204-213.

[27] Foce, F., Aita, D. (2003). The masonry arch between «limit» and «elastic» analysis. A critical re-examination of Durand-Claye's method, In: Proc. of the 1st Int. Congress on Construction History, Madrid, 20-24 January 2003, S. Huerta (Ed.), Madrid: Instituto Juan de Herrera, I, pp. 895-908.

[28] Sinopoli, A., Aita, D., Foce, F. (2007). Further remarks on the collapse mode of masonry arches with Coulomb friction, In: Proc. of $5^{\text {th }}$ Int. Conference on Arch Bridges (ARCH'07), Funchal, Madeira, Portugal, pp. 649-657.

[29] Baggio, C., Trovalusci, P. (2000). Collapse behaviour of three-dimensional brick-block systems using non-linear programming, Structural Engineering and Mechanics, 10(2), pp. 181-195.

[30] Ponterosso, P., Fishwick, R.J., Fox, D.St.J., Liu, X.L., Begg, D.W. (2000). Masonry arch collapse loads and mechanisms by heuristically seeded genetic algorithm, Computer Methods in Applied Mechanics and Engineering, 190(8-10), pp. 1233-1243.

[31] Harvey, B., Maunder, E. (2001). Thrust line analysis of complex masonry structures using spreadsheets, In: "Historical Constructions 2001 - Possibilities of Numerical and Experimental Techniques", Proc. of the 3rd Int. Seminar, Edited by Lourenço P.B. and Roca P., Guimarães, Portugal, pp. 521-528.

[32] Vermeltfoort, A.T. (2001). Analysis and experiments of masonry arches, In: "Historical Constructions 2001 Possibilities of Numerical and Experimental Techniques", Proc. of the 3rd Int. Seminar, pp. 489-498.

[33] Hughes, T.G., Hee, S.C., Soms, E. (2002). Mechanism analysis of single span masonry arch bridges using a spreadsheet, In: Proceedings of the Institution of Civil Engineers-Structures and Buildings, 152(4), pp. 341-350.

[34] De Rosa, E., Galizia, F. (2003). Evaluation of safety of pointed masonry arches through the Static Theorem of Limit Analysis, In: Proc. of 5th Int. Conference on Arch Bridges, ARCH'07, pp. 659-668.

[35] Portioli, F., Casapulla, C., Gilbert, M., Cascini, L. (2014). Limit analysis of 3D masonry block structures with non-associative frictional joints using cone programming, Computers and Structures, 143, pp. 108-121.

[36] Casapulla, C., Lauro, F. (2000). A simple computation tool for the limit-state analysis of masonry arches, In: Proc. of $5^{\text {th }}$ Int. Congress on Restoration of Architectural Heritage, pp. 2056-2064.

[37] Casapulla, C., D'Ayala, D. (2001). Lower bound approach to the limit analysis of 3D vaulted block masonry structures, In: Proc. of the $5^{\text {th }}$ Int. Symposium on Computer Methods in Structural Masonry, STRUMAS V, pp. 2836.

[38] D’Ayala, D., Casapulla, C. (2001). Limit state analysis of hemisferical domes with finite friction, In: "Historical Constructions 2001 - Possibilities of Numerical and Experimental Techniques", Proc. of the 3rd Int. Seminar, pp. 617-626.

[39] D'Ayala, D., Tomasoni, E. (2011). Three-dimensional analysis of masonry vaults using limit state analysis with finite friction, Int. J. of Architectural Heritage, 5(2), pp. 140-171.

[40] Boothby, T.E., Brown, C.B. (1992). Stability of masonry piers and arches, J. of Engineering Mechanics, ASCE, 118(2), pp. 367-383.

[41] Boothby, T.E. (1994). Stability of masonry piers and arches including sliding, J. of Engineering Mechanics, ASCE, 120(2), pp. 304-319.

[42] Boothby, T.E. (1995). Collapse modes of masonry arch bridges, Masonry International, 9(2), pp. 62-69.

[43] Smars, P. (2000). Etudes sur la stabilité des arcs et voûtes: Confrontation des méthodes de l'analyse limite aux voûtes gothique en Brabant, Doctoral Thesis, Katholieke Universiteit Leuven, Belgium, p. 229.

[44] Smars, P. (2008). Influence of friction and tensile resistance on the stability of masonry arches, In: Proc. of the $6^{\text {th }}$ Int. Conference on Structural Analysis of Historic Construction, pp. 1199-1206.

[45] Audenaert, A., Peremans, H., Reniers, G. (2007). An analytical model to determine the ultimate load on masonry arch bridges, J. of Engineering Mathematics, 59(3), pp. 323-336. 